\title{
1 Isolates from ancient permafrost help to elucidate 2 species boundaries in Acanthamoeba castellanii complex (Amoebozoa: Discosea)
}

\author{
Stas Malavin ${ }^{1}$, Lyubov Shmakova ${ }^{2}$
}

5 Institute of Physicochemical and Biological Problems in Soil Science RAS, Soil Cryology

6 Lab. Russia, 142290, Pushchino, Institutskaya 2.

$7 \quad{ }^{1}$ Corresponding author. E-mail: stas.malavin@ gmail.com

$8 \quad 2$ E-mail: lushmakova@gmail.com

\section{Abstract}

10 Acanthamoeba castellanii species complex (genotype T4) comprises of more than ten species

11 with unclear synonymy. Its molecular phylogeny has several conflicts with published

12 morphological data. In this paper, we analyze morphometric traits and temperature

13 preferences in six new strains belonging to A. castellanii complex isolated from Arctic

14 permafrost in the framework of molecular phylogeny. This integrative approach allows us to

15 cross-link genotypic and phenotypic variability and identify species-level boundaries inside

16 the complex. We also analyze previously known and newly found discrepancies between the

17 nuclear and mitochondrial gene-based phylogenies. We hypothesize that one reason for these

18 discrepancies may be the intragenomic polymorphism of ribosomal RNA genes.

19 Key words: Acanthamoeba, genotype T4, permafrost, Pleistocene, species concept, species

20 delimitation, rDNA, 18S SSU, intragenomic polymorphism, morphometrics. 


\section{Introduction}

2 Acanthamoeba is a protist genus belonging to the supergroup Amoebozoa. It has very

3 characteristic angular, or stellate, cysts with an envelope consisting of two distinct layers

4 (Fig. 1K, L, M) and fine tapered hyaline projections in the active forms - the so-called

5 acanthopodia (Fig. 1A, B, D, E). It is common in soils and aquatic environments all around

6 the world (Geisen et al., 2014). The genus is also known as an opportunistic pathogen causing

7 amoebic keratitis and encephalitis in humans (Khan, 2005; Maycock and Jayaswal, 2016).

8 Due to a well-established method of axenic cultivation, it has become a popular model in

9 biochemical and cell structure studies (Siddiqui and Khan, 2012). Acanthamoeba was among

10 the first organisms whose nuclear small subunit ribosomal RNA gene (18S rDNA) sequence

11 was determined (Gunderson and Sogin, 1986). Its genome is assembled and annotated

12 (Clarke et al., 2013), and several other draft assemblies are present in GenBank.

13 Despite such a rich history of study (or partially because of it), the system of the genus is

14 surprisingly tangled. The list of described species includes around 30 names. For several of

15 them, the synonymy is apparent from allozyme and molecular data and could also be

16 suspected for some others. Since the beginning of rDNA phylogeny era, several ribotypes

17 (traditionally called "genotypes" in Acanthamoeba literature) have been described based on a

18 threshold in genetic distances and characteristic signatures in genus-specific hypervariable

19 regions of the $18 \mathrm{~S}$ gene. These types were designated T1-T22 (Corsaro and Venditti, 2018;

20 Tice et al., 2016). While some of the types directly correspond to formally described species,

21 others include type strains of several species. This particularly concerns type T4, the most

22 frequently isolated one in both clinics and nature (Maciver et al., 2013). Internally, type T4 is

23 also heterogeneous, which has been noted since the beginning of comparative studies of the

24 18S rDNA in Acanthamoeba (Fuerst et al., 2015; Gast et al., 1996), though genetic distances 
1 between clusters inside T4 are smaller than those between this and the other types (Stothard

2 et al., 1998). Here belong the type strains of most of the species described on the basis of

3 morphology, including the generic type, Acanthamoeba castellanii (Douglas, 1930) and

4 thoroughly redescribed by Page (1967) Acanthamoeba polyphaga (Puschkarew, 1913). Due

5 to the plastic nature of characters by which T4 species are differentiated and unclear

6 synonymy, some authors prefer to designate this type "A. castellanii species complex"

7 (Corsaro et al., 2017; Kong, 2009). Therefore, reconciling molecular with morphological data

8 inside T4 is essential for the system of the genus as a whole, which means establishing a

9 genus-specific species concept integrating phenotypic and genotypic taxonomic markers

10 (Boenigk et al., 2012).

11 We isolated six new Acanthamoeba strains from Arctic permafrost sediments, a specific

12 environment, where they have been buried and trapped in a dormant but viable state. It is

13 shown that in cases when freezing occurs in parallel to sedimentation (which can be proven

14 by geological data), the age of embedded cells corresponds to the age of the permafrost

15 sediments, as both transportation and vital activity is impossible at temperatures below $10^{\circ} \mathrm{C}$

16 (Rivkina et al., 2000; Gilichinsky \& Rivkina, 2011). Our strains were isolated from the

17 sediments dated from the late Pleistocene to early Holocene. Previously, other eukaryotes,

18 including ciliates (Shatilovich et al., 2015), amoebozoans (Shmakova et al., 2016, 2018),

19 flagellates (Shatilovich et al., 2009; Stoupin et al., 2012), green algae (Vishnivetskaya, 2009),

20 fungi (Kochkina et al., 2012), and nematodes (Shatilovich et al., 2018) were isolated from the

21 sediments of similar age. Even a higher plant belonging to the genus Silene was regenerated

22 from the tissue of a seed found in the excavated borrowing of a ground squirrel, although the

23 attempts to grow those seeds failed (Yashina et al., 2012).

24 All isolated Acanthamoeba strains belong to ribotype T4. To precisely identify the taxonomic

25 position of the strains, we additionally sequenced nearly full-length $16 \mathrm{~S}$ (mitochondrial) gene 
1 and the barcode region of cytochrome oxidase subunit I (Cox1) gene. Further, we performed

2 morphometrics of all life cycle stages, including cysts formed in different conditions. We also

3 studied the growth of isolated strains at different temperatures. By integrating these data, we

4 identified a species-level boundary within the studied range of variation. Putting

5 morphometrics and ecophysiology of several strains into a broader (almost genus-wide)

6 phylogenetic framework allowed us to figure out which level of genetic heterogeneity

7 corresponds to the species-level difference in phenotypes. Additionally, we analyzed

8 previously known and newly identified discrepancies between the phylogenies based on

9 different genes and hypothesized their origin.

\section{Material and Methods}

\section{Sampling and strain isolation}

12 Samples for subsequent enrichment cultivation of amoebae were taken from the intact inner

13 part of the permafrost cores. The cores were obtained in the Gydan Peninsula (Western

14 Siberia), Kolyma Lowland, and Bykovsky Peninsula (Eastern Siberia) by a special drilling

15 technique allowing for the sterility of the sample, as described elsewhere (Gilichinskiy et al.,

16 1989; Shi et al., 1997). For the map of the borehole locations see Supplementary Fig. 1. The

17 cores were kept frozen during all steps of transportation and laboratory processing, which

18 completely prevents the contamination of the inner material by modern microorganisms (Juck

19 et al., 2005; Gilichinsky \& Rivkina, 2011).

20 Samples of about $1 \mathrm{~cm}^{3}$ were taken from the cores in a laminar flow hood using a sterile

21 scalpel and put into sterile Petri dishes filled with autoclaved mineral Prescott and James

22 medium (Page, 1988). No bacterial culture was added, as reproducing viable bacteria from

23 permafrost soil formed sufficient food supply for the emerging amoebae. The plates have 
1 been checked daily under a phase-contrast inverted microscope, and observed amoebas

2 transferred individually by a tapering Pasteur pipette into new plates, from which clonal

3 cultures were subsequently established.

\section{Culturing and encystment}

5 Strains were maintained as monoclonal bacterized cultures (the bacterial community

6 originated from initial permafrost sample) on $1.5 \%$ non-nutrient agar plates with $0,01 \%$

7 cerophyl infusion overlay (Page, 1988) at $18^{\circ} \mathrm{C}$. Cultures were passaged monthly. Cysts

8 formed in such cultures without any special induction one to two weeks after plating.

9 For axenization, cyst-rich cultures were washed from the agar layer, collected by

10 centrifugation at $1,000 \times \mathrm{g}$ for $1 \mathrm{~min}$, and treated with $1 \mathrm{M} \mathrm{HCl}$ for 24 hours. The remaining

11 cysts were then washed thrice with autoclaved distilled water and incubated in sterile

12 proteose-yeast-glucose (PYG) medium (Neff et al., 1964) with $100 \mathrm{U} \mathrm{mL}^{-1}$ of penicillin and

$130.1 \mathrm{mg} \mathrm{mL}^{-1}$ of streptomycin (Sigma-Aldrich). Axenic cultures were maintained in $25 \mathrm{~cm}^{2}$

14 culture flasks in the same medium at $18^{\circ} \mathrm{C}$ and passaged every two weeks.

15 Three conditions of encystment were tested: "bacterized culture," "sparse axenic culture,"

16 and "dense axenic culture." A typical bacterized culture represented the first. Axenic cysts

17 were obtained from cultures grown in $3 \mathrm{~mL}$ PYG on $60 \mathrm{~mm}$ plates to the monolayer stage.

18 The "sparse axenic culture" conditions were achieved as follows: a subculture of $200 \mu \mathrm{L}$ was

19 transferred into $3 \mathrm{~mL}$ Prescott and James (PJ) mineral medium (Page, 1988) and left for

20 several days. The rest of the cells were scraped and centrifuged at $1,000 \times \mathrm{g}$ for $1 \mathrm{~min}$, washed

21 with PJ, and left in $3 \mathrm{~mL}$ PJ for several days representing the "dense axenic culture"

22 conditions. From now on, we refer to the cysts formed in "bacterized culture" conditions as

23 "bacterized cysts"; to those formed in "sparse axenic culture" conditions as "sparse axenic

24 cysts"; to the cysts formed in "dense axenic culture" conditions as "dense axenic cysts." 
1 Pseudocysts were obtained from axenic cultures. Cells grown at $18^{\circ} \mathrm{C}$ in $60 \mathrm{~mm}$ Petri dishes

2 (3 mL PYG) up to the monolayer stage were washed once with PJ, collected by

3 centrifugation, and put into $3 \mathrm{~mL}$ 5\% dimethyl sulfoxide (DMSO) solution in PJ (Kliescikova

4 et al., 2011). To test the viability of the obtained pseudocysts, they were put into fresh PYG

5 medium, either immediately or after being completely dry during a week.

\section{Microscopy and measurements}

7 Living trophozoites and cysts were observed and filmed either on slides with petroleum jelly

8 sealing using Leica DM2500 microscope with phase and differential contrast optics or

9 directly in culture using Zeiss Axiostar Plus microscope with phase contrast optics and a

10 water-immersion objective. Nikon DS-Fi3 and Watec WAT-221S digital cameras were used

11 for filming in the first and the second case, correspondingly.

12 Measurements were performed on a monitor with a calibrated ruler using pictures obtained

13 with a Zeiss Axiostar Plus microscope equipped with a Watec WAT-221S camera. In the

14 case of trophozoites, locomotor forms and rounded cells were measured. The measured

15 parameters in locomotor forms included (1) the longest dimension ("the length"), (2) the

16 dimension perpendicular to the longest and going through the nucleus ("the width"), (3) the

17 diameter of the nucleus, (4) the diameter of the nucleolus, (5) the maximum width of the

18 hyaloplasm crescent at the anterior edge of the cell. For the rounded cells, cysts, and

19 pseudocysts, the diameter was registered. For the cysts, we also counted the number of

20 ostioles. The counting was done without any special staining using phase contrast optics

21 caused the ostiole complex to appear glowing, which in the case of our strains was found to

22 allow practically unambiguous identification of the ostiole number. The cyst measurements

23 were performed across all three conditions tested (see Culturing and encystment). 


\section{Statistical analysis of morphometric characters}

2 All calculations were performed in R v. 3.5.2 ( $\mathrm{R}$ Core Team, 2019). We assumed no

3 normality assumptions for all variables. For a measure of central tendency, we calculated

4 medians and expressed variation as a median absolute deviation (as implemented in package

5 stats; scale factor 1.4826) or a range. Values exceeding three interquartile range around the

6 median were removed as outliers. The statistical significance of median differences was

7 assessed with Kruskal-Wallis rank-sum test (package stats) and non-parametric

8 ("permutational") analysis of variance (PERMANOVA), as implemented in the function

9 adonis, package vegan v. 2.5-3 (Oksanen et al., 2019). Between-groups pairwise Wilcoxon

10 rank-sum tests with Benjamini \& Yekutieli correction for multiple comparisons (package

11 stats) was used for the post-hoc comparison. Clustering of the morphometric data was done

12 using the neighbor-joining algorithm on euclidean distances, as implemented in dist and

13 hclust functions.

\section{Sequence acquisition}

15 DNA was extracted from axenic cultures grown in 60-mm Petri dishes to the stationary

16 phase. Cells were scraped from the plastic and collected by centrifugation as described above.

17 The pellets were washed thrice with autoclaved distilled water and finally resuspended in 100

$18 \mu \mathrm{L}$ of it. This suspension was subjected to the DNA isolation by ExtractDNA Blood kit

19 (Proteinase K plus SDS based lysis and silica-membrane purification; Evrogen, Russia),

20 according to the manufacturer protocol.

21 The nuclear SSU rRNA gene (18S), mitochondrial SSU rRNA gene (16S), and the

22 mitochondrially encoded cytochrome c oxidase subunit I gene (Cox 1) were amplified,

23 purified by agarose gel electrophoresis, and sequenced on an automated Sanger sequencer in 
1 Evrogen Co. (Russia). Details of PCR and sequencing may be found in the Supplementary

2 Material.

3 GenBank accession numbers for the newly obtained sequences are as follows. 16S:

4 MK100243-MK100248. Cox1: MK105577-MK105582. 18S: MK124583-MK124588.

\section{Phylogenetic analysis}

6 For phylogenetic inference, all available good-quality (nearly) full-length sequences were

7 downloaded from GenBank. MAFFT v. 7.307 L-INS-i algorithm (Katoh and Standley, 2013)

8 was used to align sequences. For $18 \mathrm{~S}$, hypervariable regions of the alignment were allocated

9 into a separate partition, where gaps were coded as the fifth state (Simmons and Ochoterena,

10 2000). A phylogeny was reconstructed by Bayesian analysis of posterior probabilities

11 (Bayesian inference, BI) in MrBayes v.3.2.6 (Ronquist et al., 2012) and maximum likelihood

12 (ML) approach in IQTree v.1.6.6 (Nguyen et al., 2015). The detailed description of the

13 phylogenetic analyses may be found in Supplementary Material.

\section{Growth at different temperatures}

15 We tested the growth of the isolated strains at $4^{\circ} \mathrm{C}, 11^{\circ} \mathrm{C}, 18^{\circ} \mathrm{C}, 25^{\circ} \mathrm{C}, 32^{\circ} \mathrm{C}, 37^{\circ} \mathrm{C}, 40^{\circ} \mathrm{C}$, and

$1642^{\circ} \mathrm{C}\left(\Delta=1{ }^{\circ} \mathrm{C}\right.$ for all $)$. At $4-32^{\circ} \mathrm{C}$, only axenic cultures were tested, while to $37-42^{\circ} \mathrm{C}$, both

17 axenic and bacterized cultures were exposed. Preliminarily, all temperatures were checked

18 with 40-mm plates ( $1 \mathrm{~mL}$ PYG) in several replicates, to test if growth is possible at all.

19 Axenic cultures for the tests were obtained by transferring $50 \mu \mathrm{L}$ of a 5-day culture. In the

20 case of bacterized cultures, test plates were inoculated with $50 \mu \mathrm{L}$ of a two-week culture. This

21 assay was carried out for one week; the cultures were checked daily.

22 For the temperatures, at which the strains have demonstrated growth or at least persisted

23 successfully, the growth rate was estimated quantitatively. 12-well culture plates, $2 \mathrm{~mL}$ of 
1 PYG per well, were inoculated with a growing axenic culture as described above. Each strain

2 was tested in two to three replicates per temperature level. The assays were conducted until

3 detached rounded cells started to prevail in the culture. Cells were counted in a

4 hemocytometer each $24 \mathrm{~h}$ (each $12 \mathrm{~h}$ at $32^{\circ} \mathrm{C}$ ). At $4^{\circ} \mathrm{C}$, the experiment lasted for a month, and

5 samples for counting were taken weekly, as observations showed no discernible increase of

6 the cell number. The culture growth was approximated by a logistic curve $N=\frac{L}{1+e^{-k\left(x-x_{0}\right)}}$,

7 where $L$ is the asymptote and $k$ - the logistic growth rate. The approximation was done by

8 Levenberg-Marquardt nonlinear least-squares algorithm as implemented in MINIPACK

9 library interfaced through minpack.lm package v.1.2-1 (Elzhov et al., 2016) for R v.3.5.2.

\section{Results}

\section{Isolated strains}

12 In total, we have obtained ten Acanthamoeba isolates from around 400 studied permafrost

13 sediment samples from East and West Siberia. We have succeeded in cloning and axenization

14 of six out of ten isolates. The location of the sampling sites is shown in Supplementary Fig. 1.

15 For the essential characteristics of the samples, see Tab. 1.

\section{Microscopy observations}

17 All obtained strains feature typical morphological characters of the genus, i.e., acanthopodia

18 in trophozoites and angular shape of the endocyst, and belong to the morphotype II following

19 Pussard and Pons (1977).

20 Trophozoites

21 Trophozoites of all strains readily develop locomotor forms when transferred to fresh 
1 medium or the slide. The locomotor form is flattened, with a distinct frontal hyaline area

2 usually occupying about one-fourth of the cell length (Fig. 1A-E). Typically, it is elongated

3 in the direction of movement, with a width-to-length ratio of about 0.6 (Fig. 1A, B).

4 Occasionally, we have observed locomotor forms with the width equal to or even greater than

5 length (Fig. 1C, D). Such forms have been encountered more often in strains SCL-14-2, SCL-

6 14-3, SCL-14-12, and are generally more typical for larger cells. Acanthopodia are absent on

7 the leading lobopodium in locomotor forms, though the hyaline crescent edge has rarely been

8 even for a long time, regularly demonstrating undulations and protrusions. In cells moving

9 irregularly, acanthopodia are present on lobopodia also. At the beginning of an active

10 movement or while changing its way, trophozoites demonstrate sudden extensions of the

11 lobopodium in a new direction similar to the eruptive movements of heterolobose amoebae.

12 Generally, one vesicular nucleus with a single nucleolus is situated in the central part of the

13 cell in locomotor forms. At temperatures 25 to $32{ }^{\circ} \mathrm{C}$, we have observed multinucleate

14 trophozoites (Fig. 1G). Those have always been bigger than the median, while nucleoli have

15 been smaller than in normal cells (Fig. 1G).

16 A single contractile vacuole is usually situated in the rear part of the moving cell (Fig. 1A,

17 B). In axenic cultures, the cytoplasm is strongly vacuolated, while in bacterized cultures, the

18 number of vacuoles is found to be considerably lower. A bulbous uroid often appeared in

19 locomotor forms (Fig. 1A, C, D), sometimes with adhesive filaments (Fig. 1D). Sporadically,

20 we observed a sizeable goblet-like food cup at the rear end of the moving cell (Fig. 1E),

21 identically to what was described and figured by Page (1967). This formation was

22 considerably more often found in strain SCL-14-12.

23 Floating forms have been rarely observed in bacterized cultures. The floating form represents

24 a central body mass with 15-20 radiating pseudopodia up to $15 \mu \mathrm{m}$ long (Fig. 1F). 
1 In axenic cultures grown in plates or culture flasks without agitation, amoebae form a

2 characteristic monolayer on the surface of the substrate (Fig. 1H). The "closeness" of the

3 monolayer depends on the temperature and adhesive properties of the plastic and differs

4 across the vessel area (Fig. 1H demonstrates the "ideal" case). After the monolayer reaches

5 its closeness limit, cells start to appear above it massively. This scenario is followed by all

6 studied strains but SCL-14-12, in which cells begin to form compact clumps above the

7 surface from the early stages of the culture growth, in parallel to the monolayer formation.

8 When the culture is roughly shaken or centrifuged, trophozoites appear as rounded cells

9 without lobopodia and acanthopodia. Such cells demonstrate the internal movement of

10 organelles, though no cell movements can be observed for some time. Similar rounded cells

11 also start to appear soon after an axenic culture reaches its growth limit. In this case, most of

12 them probably die in some time, as the final number of cysts in such cultures is much lower

13 than the number of rounded cells observed at the end of growth.

\section{Cysts}

15 All isolated strains readily encyst in laboratory cultures. In bacterized cultures, all or at least

16 most of the amoebae form cysts in about two weeks after transfer. In axenic cultures, we

17 obtained cysts by transferring amoebae into either the Neff's encystment medium (Neff et al.,

18 1964) or merely starving PJ medium. When starting to encyst, cells form plaques.

19 Mature cysts of all strains have well-defined endo- and ectocyst, the former folded and

20 rippled, the latter smoothly polyhedral. Beneath the endocyst, the characteristic layer of

21 oriented granules is always apparent (Fig. 1I, K, L, M). The number of endocyst corners

22 usually vary from 3 to 7 (Fig. 1I, K, M, O), but cysts with 8 and more corners have also been

23 encountered (Fig. 1L). The majority of ostioles lay in one plane. Opperculi do not protrude

24 above the ectocyst level. Dense axenic cysts usually have less prominent endocyst corners 
1 than sparse axenic cysts and bacterized culture cysts. In the latter two, a considerable share of

2 cysts has smooth endocyst without any defined corners (Fig. 1I).

\section{Pseudocysts}

4 The pseudocyst formation is total only at DMSO concentrations not lower than $5 \%$ and when

5 the DMSO-to-cells ratio is not less than the PYG-to-cells ratio during culture growth. In such

6 conditions, all cells transform into pseudocysts in 12 hours. Both dried and non-dried

7 pseudocysts demonstrate almost total viability being put into PYG; in both cases,

8 trophozoites appear in no more than a day.

9 Pseudocysts are rounded cells with a fine smooth envelope well seen under both phase- and

10 DIC-contrast (Fig. 1J). The nucleus is situated in the central area of the cell; the nucleolus is

11 slightly smaller than in trophozoites. The cytoplasm is evenly filled with refractile granules.

12 The size of the granules in pseudocysts is bigger than that in cysts. No granular layer beneath

13 the envelope characteristic for cysts may be observed.

\section{Molecular phylogeny}

\section{$1518 \mathrm{~S}$}

16 The inspection of the preliminary automatic alignment of the T4 $18 \mathrm{~S}$ sequences has revealed

17 six hypervariable regions corresponding to the parts of the third, fifth, sixth, ninth, and

18 eleventh hypervariable (“expansion”) regions identified by Gast et al. (1996). Two of those

19 six regions are parts of the diagnostic fragment 3 (DF3) (Booton et al., 2002). The DF3

20 region is used to distinguish sequence types (Booton et al., 2009, 2005, 2002), but we have

21 found it sufficient to discriminate between T4 subtypes also, except for some rare cases of

22 closely related sequences. The alignment of concatenated hypervariable regions has yielded

23 easily discernible patterns (Supplementary Fig. 3) and has, first of all, demonstrated many 
1 several-nucleotide-long indels present in many sequences which by any chance could not be

2 attributed to the alignment method.

3 Both $\mathrm{BI}$ and $\mathrm{ML}$ analyses produce seven subtypes, $\mathrm{T}_{\mathrm{A}}$ through $\mathrm{T} 4_{\mathrm{E}}$ and $\mathrm{T} 4_{\mathrm{Neff}}$, previously

4 mentioned by Fuerst (2014) and Fuerst et al. (2015), further referred to as A-E and Neff. All

5 but $\mathrm{A}$ and $\mathrm{B}$ receive maximum support in both analyses; B is highly supported (0.9) and A

6 moderately supported (0.78) in the BI analysis. At the same time, the ML and BI trees display

7 one difference in their topologies (Fig. 2). While in both trees the major division lay between

8 the clades $\mathrm{A}+\mathrm{B}$ and $\mathrm{C}+\mathrm{F}+\mathrm{E}$, in the ML tree, subtype $\mathrm{D}$ appear sister to the $\mathrm{A}+\mathrm{B}$ clade and

9 subtype Neff sister to subtype E. In the BI tree, vice versa, subtype D is sister to subtype E

10 and subtype Neff sister to the A+B clade.

11 Several subclusters have got high support in both ML and BI analyses inside clades A and B

12 (Fig. 2). From now on, we refer to them using well-known and long-studied strains they

13 include, for which $16 \mathrm{~S}$ sequences are also available. This is done for the sake of

14 descriptiveness and does not imply that these strains are somehow "characteristic" of the

15 clusters being described. In clade A, those are "Castellani," "Galka," "Haas," "Rawdon,"

16 "Garcia" clades, and one other, not discussed further. In clade B, those are "Ma," "Diamond,"

17 "V017" clades, and one other.

18 In $\mathrm{T} 4_{\mathrm{A}}$, the topology of the Bayesian tree differs from that of the ML tree (Fig. 3) chiefly by

19 the position of "Haas" clade, which is sister to the cluster containing "Galka" and

20 "Castellani" clades instead of being the deepest $\mathrm{T} 4_{\mathrm{A}}$ branch as in the ML tree, and the

21 position of "Garcia" clade placed inside "Rawdon" clade instead of being the second deepest

22 branch as in the ML tree.

23 Strains SCL-am8, SCL-14-2, and SCL-14-3 branch together in the ML tree (Fig. 3) and

24 group with strain COHH 43 from marine sediments of Mt Hope Bay, MA, USA (Gast et al., 
1 2011) as a distinct clade sister to "Ma" clade of T4в. In the BI tree, the permafrost strains and

2 COHH 43 branch before "Ma" clade. Other differences between the BI and ML tree

3 topologies in $\mathrm{T}_{\mathrm{B}}$ involved terminal branching only. Three other permafrost strains grouped

4 within T4 $\mathrm{A}$ clade. Strain SCL-14-9 is relatively distant from the other strains except for one

5 clinical isolate KA/E6 from Korea (Liu et al., 2006); their position in the tree is unstable.

6 Strain SCL-am9 is firmly placed into "Galka" clade, and strain SCL-14-12 - into "Castellani"

7 clade.

$816 \mathrm{~S}$

9 The analysis of the $16 \mathrm{~S}$ alignment confidently recovers all the previously established $18 \mathrm{~S}$

10 types for which $16 \mathrm{~S}$ sequences are available (Fig. 4). We thus refer to them further without

11 mentioning in the framework of which analysis are they inferred. Type T4 is moderately

12 supported (94\%) in ML-analysis but has posterior probability of 1 in BI. T2/6 type is sister to

13 T4 type; the next-closest is T5 followed by T3/T11 clade, though this topology as a whole

14 appears moderately supported. Types T7-T9 (morphogroup I by Pussard and Pons (1977)

15 form a very distinct clade with full support.

16 The internal structure of 16S-T4 clade partially reflects that of 18S-T4. D, E, and Neff

17 subtypes appear monophyletic with full support. At the same time, subtypes A and B, as well

18 as $\mathrm{C}$ and $\mathrm{F}$, are not separate. Subtype $\mathrm{E}$ is the most distant from A/B clade, followed by C/F

19 clade, then subtype Neff. Subtype D is the closest to A/B clade. The $16 \mathrm{~S}$ tree thus

20 corroborates the $18 \mathrm{~S}$ one inferred with the ML approach, besides the $\mathrm{A} / \mathrm{B}$ and $\mathrm{C} / \mathrm{F}$ fusion.

21 The blended 16S A/B clade, like its 18S separate counterparts, is internally structured

22 (Fig. 4). Galka, Rawdon, and Garcia strains from 18S-T4A also hit highly supported clusters.

23 Strain Haas does not fall into any cluster but is itself steadily separated. At the same time,

24 strains Castellani and Ma have very similar 16S sequences and form a supported cluster, 
1 together with some other strains. The same concerns strains Diamond and CDC:V017.

2 Surprisingly, all strains from permafrost except SCL-14-12 have identical 16S sequences,

3 also identical to those of strains AC11 and AC16 from India (Megha et al., 2018). In addition

4 to AC15, AC20, AC28, and CDC:V168 (18S-T4A $)$, they form a highly-supported cluster

5 inside T4. SCL-14-12 groups together with Indian AC4 (Megha et al., 2018) and JPH24 from

6 Japan (Rahman et al., 2013) (18S-T4B) as a sister clade to a group containing strains Galka,

7 HC-2, CDC:V390, Liu-E1 (all 18S-T4A), with absolute support. Indian strains AC13 and

8 AC25 reveal rather divergent sequences, probably corresponding to a not yet recovered T4

9 subtype. Unfortunately, their $18 \mathrm{~S}$ sequences in GenBank are not full, so it is not possible to

10 either confirm or deny this hypothesis by now.

\section{Cox 1}

12 As with the $16 \mathrm{~S}$ gene, strains belonging to Rns D, E, and Neff subtypes fall into different

13 highly supported clusters, while those within Rns A and B, as well as C and F, form fused

14 clusters (Fig. 5). Clade D groups together with clades C/F, E, and Neff. This subdivision is

15 not supported by ML analysis, though gained 0.97 posterior probability. Inside the C/F/Neff

16 half of the tree, no subtype relationships are confidently resolved.

17 As with 16S, all permafrost strains, except SCL-14-12, possess identical sequences. Together,

18 they form a supported clade with three sequences from the Ohio State University collection

19 (OSU) 07-033, 07-003 (unfortunately, lacking available 18S sequences) and 07-027 (18S-

$20 \mathrm{~T}_{\mathrm{A}}$ ). A sequence of unknown strain identified as Acanthamoeba quina Pussard et Pons, 1977

21 (genome CDFN) appears sister to that clade with high support. Another highly supported

22 cluster, forming a sister to the just described clade, contains the sequence of strain V017 (Rns

$23 \mathrm{~T} 4_{\mathrm{B}}$ ), sequences from genomes CDFL, CDFK, and CDFJ (probably misidentified as

24 Acanthamoeba pearcei Nerad, Sawyer, Lewis et McLaughlin, 1993), and a set of OSU 
1 sequences belonging to A and B 18S subtypes. Permafrost strain SCL-14-12 groups together

2 with strains OSU 06-024 and 08-016 (both 18S-T4A). One more firm cluster includes the

3 sequence from genome CDFB, identified as Acanthamoeba lugdunensis Pussard et Pons,

4 1977. Other strains in this cluster are Linc Ap-1 (genome LQHA) and two OSU strains, both

5 attributed to $18 \mathrm{~S}-\mathrm{T} 4_{\mathrm{A}}$. The last cluster in $\mathrm{T} 4_{\mathrm{A} / \mathrm{B}}$ is that encompassing sequences of strain

6 LVPEI 1060/96 (18S-T4 $\left.4_{\mathrm{B}}\right)$ together with OSU sequences 02-031 (18S-T4 $\left.\mathrm{A}\right), 05-023$ and 07-

$7019(18 \mathrm{~S}-\mathrm{T} 4 \mathrm{~B})$.

\section{Morphometrics}

\section{Trophozoites}

10 The length of the measured trophozoites of all strains is between 15 and $47 \mu \mathrm{m}$, and the width

11 between 5 and $27 \mu \mathrm{m}$ (Fig. 6, Tab. 2). Locomotor forms of strain SCL-14-12 are considerably

12 shorter (median $23 \mu \mathrm{m} ; \square X^{2}(1,5)=92.765, p<2.2 \times 10^{-16}$ ). The difference in length in all

13 other strains is not significant. At the same time, the most narrow locomotor forms are those

14 of strain SCL-am9 (median $12 \mu \mathrm{m} ; \square X^{2}(1,5)=51.785, p=5.971 \times 10^{-10}$ ). This leads to the

15 significant difference in width-to-length ratio in the two strains, which is the biggest in SCL-

$1614-12$ (median 0.69) and the smallest in SCL-am9 (median 0.46) $\left(X^{2}(1,5)=72.762, p=\right.$

$\left.172.725 \times 10^{-14}\right)$. This distinction in shape could be clearly observed by eye when inspecting a

18 series of locomotor forms (Fig. $1 \mathrm{~A}, \mathrm{~B}, \mathrm{C}$ demonstrates the typical variants).

19 Some slight differences in the diameter of the nucleus and nucleolus have been observed. The

20 median nucleus diameter in locomotor forms of strains SCL-14-12 and SCL-am9 $(4 \mu \mathrm{m})$ are

21 slightly lower than in other strains $(5 \mu \mathrm{m})\left(\square X^{2}(1,5)=62.971, p=2.952 \times 10^{-12}\right)$. The

22 difference is marginally significant between SCL-14-12 and SCL-14-9, SCL-am9 and SCL-

23 am8, and not significant between SCL-14-12 and SCL-am8. At the same time, the nucleolus

24 in SCL-14-12 is significantly bigger (median diameter $3 \mu \mathrm{m})$ than in other strains $(2 \mu \mathrm{m})$ 
$1 \quad\left(X^{2}(1,5)=46.089, p=8.712 \times 10^{-9}\right)$. This results in significantly higher nucleolus-to-nucleus

2 diameter ratio in strains SCL-14-12 and SCL-am9 (not different from SCL-14-9), and also

3 partially in strain SCL-14-9. In strain SCL-14-12, the median of the hyaline crescent breadth

4 is the lowest (4 $\mu \mathrm{m})$ differing significantly from all other strains $\left(\square X^{2}(1,5)=128.58, p<\right.$

$\left.52.2 \times 10^{-16}\right)$. Strain SCL-14-9 has the next lowest breadth, also differing significantly from

6 other strains at 0.05 level.

7 Rounded cell diameter is between $15 \mu \mathrm{m}$ in SCL-14-12 and $19 \mu \mathrm{m}$ in SCL-14-9 and well

8 correlates to the cell length (Spearman's $\rho=0.87, S=4.6324, p=0.025$ ).

9 Cysts

10 We have found the cyst diameter a good character in terms of dispersion and inter-group

11 differences. Not surprisingly, the dispersion of the cyst diameter in all estimated factor

12 groups ("Strains" per "Culture type" design) is lower than that of the length and width in

13 locomotor forms (MAD not greater than $12 \%$ of the median). The range of the measured cyst

14 diameters is 10 to $24 \mu \mathrm{m}$ (medians from 13 to $17 \mu \mathrm{m}$ in bacterized cultures, from 14 to $17 \mu \mathrm{m}$

15 in dense axenic cultures, and 15 to $19 \mu \mathrm{m}$ in sparse axenic cultures) (Tab. 3). Permutational

16 analysis of variance shows the significance of the effect of "Strain" $(F(1,5)=263.6$,

$17 p<.001)$, "Culture type" $(F(1,2)=420.4, p<.001)$, and their interaction $(F(1,10)=29.09$,

$18 p<.001)$. Generally, the cysts formed in bacterized cultures are the smallest followed by the

19 cysts from dense axenic cultures and then by the cysts from sparse axenic cultures, which in

20 all strains are about the same size than the "normal" rounded trophozoites (Fig. 7), indicating

21 almost no shrinkage during the cyst formation. In strains SCL-am8 and SCL-14-9, these

22 differences in diameter are the most prominent, while SCL-14-12 has the smallest range (the

23 difference is still significant).

24 No variation in the number of operculi is observed between cysts formed in different 
1 conditions.

\section{Pseudocysts}

3 The diameter of the pseudocysts in the studied strains varies from 11 to $16 \mu \mathrm{m}$ (Tab. 4). As

4 with cysts, SCL-14-12 is the smallest. Also, in all strains except SCL-14-9 and SCL-am8,

5 pseudocysts are smaller than any type of cysts. In the two mentioned strains, cysts formed in

6 bacterized cultures are slightly but significantly smaller than pseudocysts (Fig. 7).

\section{Growth at different temperatures}

8 The results of the preliminary test demonstrate that none of the isolated strains can maintain

9 the stable growth at temperatures equal to or higher than $37^{\circ} \mathrm{C}$, whether as axenic cultures in

10 PYG medium or bacterized cultures on agar plates with WG medium overlay. At 40 and

$1142^{\circ} \mathrm{C}$, cells die entirely in about a day. At $37^{\circ} \mathrm{C}$, some cells remain alive for several days

12 (more in bacterized than in axenic cultures), but their number declined to zero to the end of

13 the week.

$1432^{\circ} \mathrm{C}$ is not favorable for all cultures, although at this temperature, they exhibit the fastest

15 growth (Fig. 8, Tab. 5). In strains SCL-am8, SCL-am9, SCL-14-2, and SCL-14-3, cells do

16 not attach to the substratum and always appear roundish. A typical monolayer has never been

17 observed. Multinucleate cells start to appear from the first counting; subsequently, their

18 amount and size increase. The population density curve reaches stationarity very quickly. The

19 maximum culture density at this temperature does not exceed $8 \times 10^{5}$ cells $\mathrm{mL}^{-1}$ for SCL-am8,

$205 \times 10^{5}$ cells $\mathrm{mL}^{-1}$ for SCL-14-2 and SCL-14-2, $2 \times 10^{5}$ cells $\mathrm{mL}^{-1}$ for SCL-am9. Strain SCL-

21 14-12 attaches to the bottom and grows better than all in terms of the culture density (up to

$222 \times 10^{6}$ cells $\mathrm{mL}^{-1}$ have been recorded), though this constitutes a considerable decrease

23 compared to the densities at lower temperatures. Strain SCL-14-9 demonstrates a behavior 
1 somewhat intermediate between SCL-14-12 and others. During the first two days, the culture

2 grows well, and cells attach to the substratum. Later, most of them are found roundish and

3 detached, and the monolayer is never formed. Multinucleate cells also appear, but later than

4 in others and lower quantity. The density reaches $6 \times 10^{5}$ cells $\mathrm{mL}^{-1}$.

5 At $25^{\circ} \mathrm{C}$, all strains grow well and form a monolayer in three to four days. Generally, the

6 culture density increases faster than at $18^{\circ} \mathrm{C}$ but stops growing earlier, reaching the lower

7 maximum. In strains SCL-am8, SCL-14-2, SCL-14-3 multinucleate cells also appear.

$818^{\circ} \mathrm{C}$ is the optimal temperature for all strains. The maximal recorded densities are up to

$98 \times 10^{5}$ cells $\mathrm{mL}^{-1}$ in SCL-14-2 and SCL-14-3; $1.5 \times 10^{6}$ cells $\mathrm{mL}^{-1}$ in SCL-am8, SCL-am9, and

$10 \mathrm{SCL}-14-9 ; 2.8 \times 10^{6}$ cells $\mathrm{mL}^{-1}$ in SCL-14-12) since the active growth continues longer,

11 although the growth rate is slightly lower than at $25^{\circ} \mathrm{C}$. Cultures form a monolayer in four to

12 seven days. Cells form pseudopodia and actively move during a long time, even at the

13 stationary phase of growth.

14 At $11^{\circ} \mathrm{C}$, all the cultures demonstrate a considerably lower rate of growth (Fig. 4). SCL-14-12

15 reaches a plateau in about $600 \mathrm{~h}$ at a density of more than $3 \times 10^{6}$ cells $\mathrm{mL}^{-1}$. Other strains

16 show similar dynamics and reach a plateau in $400 \mathrm{~h}$ at a density of about $1.5 \times 10^{6}$ cells $\mathrm{mL}^{-1}$

17 in SCL-am8 and about $1.0 \times 10^{6}$ cells $\mathrm{mL}^{-1}$ in others.

18 At $4{ }^{\circ} \mathrm{C}$, all the cultures have been in a good state during the whole period of observation,

19 judging by the cell and overall culture appearance. However, counting has shown that the cell

20 number has been slightly declining, although dividing cells have been observed during the

21 first two weeks.

22 The modeling of the logistic growth demonstrates three differences of SCL-14-12 in

23 comparison to other strains. First, its density asymptote is higher than in other strains at any 
1 tested temperature, including $32^{\circ} \mathrm{C}$. Second, its growth rate is about the same at 18 and $25^{\circ} \mathrm{C}$,

2 whereas in other strains, the growth rate at $25^{\circ} \mathrm{C}$ is considerably higher than at $18^{\circ} \mathrm{C}$.

3 Consequently, at $25^{\circ} \mathrm{C}$, the growth rate of SCL-14-12 is lower than that of other strains. At

4 the same time, at $32^{\circ} \mathrm{C}$, the growth rate of SCL-14-12 is strikingly higher, while in others it is

5 lower, the same, or (in SCL-14-3) just a little bit higher than at $25^{\circ} \mathrm{C}$.

\section{Discussion}

\section{Molecular phylogeny of $A$. castellanii complex (type T4)}

$8 \quad 18 S$

9 The distinctiveness of different clades inside type T4 has begun to manifest itself since the

10 first works on Acanthamoeba rDNA diversity (Booton et al., 2002; Chung et al., 1998; Fuerst

11 et al., 2015; Gast et al., 1996; Kong, 2009; Martín-Pérez et al., 2017; Stothard et al., 1998).

12 Subtypes $\mathrm{T} 4_{C}, T 4_{D}, T 4_{E}, T 4_{F}$, and $\mathrm{T} 4_{N e f f}$, when represented by several sequences, were

13 usually well-supported, while $\mathrm{T} 4_{\mathrm{B}}$ was sometimes mixed with $\mathrm{T} 4_{\mathrm{A}}$, and the latter mostly

14 paraphyletic. At the same time, the relationship between the subtypes was never well-

15 supported and appeared in part unstable. The deepest branching clade was either $\mathrm{T} 4_{\mathrm{D}}, \mathrm{T} 4_{\mathrm{E}}$, or

16 a clade containing both of them. $\mathrm{T} 4_{\mathrm{Neff}}$ appeared sister to either $\mathrm{T} 4_{\mathrm{A} / \mathrm{B}}$ or $\mathrm{T} 4_{\mathrm{D} / \mathrm{E}}$ mixed clades.

$17 \mathrm{~T} 4_{\mathrm{C} / \mathrm{F}}$ clade always branched after $\mathrm{T} 4_{\mathrm{D} / \mathrm{E}}$, except for the phylogeny using GTR model, where

$18 \mathrm{~T}_{\mathrm{D}}$ was robustly sister to $\mathrm{T} 4_{\mathrm{A} / \mathrm{B} / \mathrm{Neff}}$ cluster (Martín-Pérez et al., 2017).

19 In our case, automatic alignment with subsequent filtering yields robust clades (subtypes)

$20 \mathrm{~T}_{\mathrm{C}}$ through $\mathrm{T} 4_{\mathrm{Neff}}$, moderately supported $\mathrm{T} 4_{\mathrm{B}}$, and paraphyletic $\mathrm{T} 4_{\mathrm{A}}$ (tree not shown). All

21 seven subtypes as monophyletic clades could only be obtained with Jukes-Cantor substitution

22 model, the least-likely one in MrModelTest assessment. Relationships between the subtypes 
1 are poorly supported. However, the fifth-state-coding approach demonstrates the

2 distinctiveness of all the $\mathrm{T} 4$ subtypes. The fact that $\mathrm{T} 4_{\mathrm{C}}-\mathrm{T} 4_{\mathrm{F}}$ subtypes may be obtained from

3 the masked alignment demonstrates the adequateness of the fifth-state-coding method used.

4 Moreover, monophyletic and fully-supported $\mathrm{T} 4_{\mathrm{B}}, \mathrm{T} 4_{\mathrm{D}}-\mathrm{T} 4_{\mathrm{F}}$, and $\mathrm{T} 4_{\mathrm{Neff}}$ may also be

5 obtained using the alignment of conservative regions only.

6 Thus, our $18 \mathrm{~S}$ phylogeny corroborates and validates the previously obtained results. At the

7 same time, the method bears some possible sources of bias: (i) Indels of more than one

8 nucleotide are treated as separate events, while they may actually represent a single event, (ii)

9 Inversions are treated as several events, (iii) The amount of transversions is probably

10 underestimated, (iv) Unresolvable position of indels in single-nucleotide repeats. This

11 probable bias may explain differences in topology between BA and ML trees.

$1216 \mathrm{~S}$

13 As demonstrated by Ledee et al. (2003), 16S and 18S-based phylogenies of Acanthamoeba

14 corroborate each other in terms of identified sequence types (ribotypes). This holds for T4

15 type as well. Further, even a restriction analysis of the 16S gene (Byers et al., 1983) has

16 revealed remarkable heterogeneity inside $16 \mathrm{~S}-\mathrm{T} 4$ comparable to that found in 18S-T4. At the

17 same time, this study has first discovered the major inconsistency between $18 \mathrm{~S}$ and $16 \mathrm{~S}$

18 phylogenies - Castellani and Ma strains $\left(18 \mathrm{~S}-\mathrm{T} 4_{\mathrm{A}}\right.$ and $\mathrm{T} 4_{\mathrm{B}}$, correspondingly) appeared

19 closely related and showed differences at the level of intraspecific variation in other

20 organisms. Other 18S-T4 type strains included in this study, namely Neff, Singh, and Page-

2123 , were found to be as distant as other ribotypes.

22 The sequence-based 16S phylogeny (Ledee et al., 2003) demonstrated the identity of

23 Castellani and Ma (as well as CDC:V042) sequences. Here, about $15 \%$ bases were removed

24 as ambiguously aligned, which explains why differences found by Byers et al. (1983) were 
1 not observed. The rest of the sequences attributed to $18 \mathrm{~S}-\mathrm{T} 4_{\mathrm{A}}$ and $\mathrm{T} 4_{\mathrm{B}}$ subtypes were

2 intermixed. Nine new clades were identified inside 16S-T4. Sequences of the strains

3 attributed to $18 \mathrm{~S}$ subtypes $\mathrm{T}_{\mathrm{C}}, \mathrm{T} 4_{\mathrm{D}}, \mathrm{T} 4_{\mathrm{E}}, \mathrm{T} 4_{\mathrm{Neff}}$ formed corresponding clades on the $16 \mathrm{~S}$ tree

4 (T4 $4_{\mathrm{F}}$ sequences were not included). The deepest branching clade was $\mathrm{T} 4_{\mathrm{E}}$ following by $\mathrm{T} 4_{\mathrm{C}}$,

5 then $T 4_{\text {Neff }}$ clade. $T 4_{D}$ sequences formed a clade sister to mixed T4 $4_{\mathrm{A} / \mathrm{B}}$ clade. Kong (2009)

6 using a smaller dataset identified five $16 \mathrm{~S}$ clades, namely the deepest branching $\mathrm{T}_{\mathrm{C}}$, the next

7 branching $\mathrm{T} 4_{\mathrm{D}}$, then $\mathrm{T} 4_{\mathrm{Neff}}$, and the last branching $\mathrm{T} 4_{\mathrm{A} / \mathrm{B}}$, which in turn consisted of two

8 clusters: the one containing, among others, Ma and Castellani strains and another one

9 containing A. lugdunensis L3a. Finally, Rahman et al. (2013) adding 13 T4 rns sequences

10 obtained generally the same nine clades as Ledee et al. (2003) inside T4 subtype.

11 We have identified around 13 well-supported clusters inside 16S-T4 subtype. In addition to

12 the proximity of Castellani and Ma strains, our study reveals other discrepancies between the

$1318 \mathrm{~S}$ and 16S phylogenies. Permafrost strains SCL-am9 and SCL-14-9 belonging to 18S-T4

14 subtype and SCL-am8, SCL-14-2, SCL-14-3, belonging to 18S-T4 ${ }_{\mathrm{B}}$ subtype, all have

15 identical 16S sequences (even before alignment masking). Strains CDC:V042 (ATCC 50493)

16 and CDC:V390 get into same "Rawdon" clade in 18S tree, but appear all distant in 16S tree.

17 Strain CDC:V042 is close to Castellani strain while being placed in a different highly

18 supported subcluster of T4A by $18 \mathrm{~S}$ phylogeny. Galka strain (ATCC 50496) groups together

19 with SCL-am9 on the $18 \mathrm{~S}$ tree, but is steadily apart from it on the $16 \mathrm{~S}$ tree. By contrast, strain

20 CDC:V390 robustly branches apart from Galka on the $18 \mathrm{~S}$ tree but has identical $16 \mathrm{~S}$

21 sequence. At the same time, strain Liu-E1 (ATCC 50709) is close to Galka in terms of 18S,

22 and 16S sequences of these two strains are identical too. Strain SCL-14-12, which robustly

23 clusters together with Castellani strain in absolutely all $18 \mathrm{~S}$ analyses, steadily falls apart from

24 it on the 16S tree. Finally, strains Rawdon (BCM:37, ATCC 50497) and CDC:V390 cluster

25 together with high support on the $18 \mathrm{~S}$ tree, but are steadily apart on the $16 \mathrm{~S}$ tree. 
1 To summarize, some of the strains demonstrate proximity of their $18 \mathrm{~S}$ sequences and have

2 distant $16 \mathrm{~S}$ sequences, some, vice versa, have distant $18 \mathrm{~S}$ sequences and similar $16 \mathrm{~S}$

3 sequences, and other ones demonstrate similarity in both. To explain such complex

4 dissimilarity between $18 \mathrm{~S}$ and $16 \mathrm{~S}$ phylogenies, three hypotheses may be involved. The first

5 implies different rates of gene evolution in different strains, which is unlikely regarding their

6 taxonomic proximity. As the second, the improper alignment of indels may be invoked (see

7 the consideration of possible sources of bias above). Indeed, in single-nucleotide stretches in

8 an alignment, indels may occur in any position and ideally should not be aligned (which is

9 nonetheless done for the sake of parsimony). When indels are treated as the fifth state and

10 included in the calculation, their alignment may force sequences to appear more similar than

11 they are. This possibility cannot be completely ruled out here, but against it is the fact that

12 only A and B and C and F 18 S subtypes became intermingled in $16 \mathrm{~S}$ tree, while the others

13 (D, E, and Neff) stayed separate. Also, this hypothesis may explain why sequences that are

14 close to each other in $18 \mathrm{~S}$ phylogeny appear distant in $16 \mathrm{~S}$ one, but not the opposite cases (as

15 our 16S phylogeny did not account for indels).

16 The third hypothesis maintains that $18 \mathrm{~S}$ subtypes $\mathrm{T} 4_{\mathrm{A}}$ and $\mathrm{T} 4_{\mathrm{B}}$, as well as $\mathrm{T} 4_{\mathrm{C}}$ and $\mathrm{T} 4_{\mathrm{F}}$,

17 actually represent intra-genomic variants of the gene present in the cell in a different number

18 of copies, with only the dominant one revealed by the PCR. Different strains may bear

19 different dominant variants and thus be attributed to either $\mathrm{A}$ or $\mathrm{B}(\mathrm{C}$ or $\mathrm{F})$ subtype while

20 sharing the same (or closely related) $16 \mathrm{~S}$ gene sequences. Intragenomic variation in $18 \mathrm{~S}$ gene

21 sequence is known in different protist supergroups including other Amoebozoa (Amaral-

22 Zettler et al., 2006; Gong et al., 2013; Gribble and Anderson, 2007; Kudryavtsev and

23 Gladkikh, 2017; Nassonova et al., 2010; Ntakou et al., 2019; Pillet et al., 2012) and reported

24 for several Acanthamoeba strains (Nuprasert et al., 2010; Qvarnstrom et al., 2013; Rahman et

25 al., 2013; Stothard et al., 1998). Furthermore, among 18S intragenomic variants found by 
1 Rahman et al. (2013) in two strains, one belongs to $\mathrm{T}_{\mathrm{A}}$ and another to $\mathrm{T} 4_{\mathrm{B}}$ (the alignment is

2 available upon request). Also, this hypothesis may explain common difficulties with

3 obtaining a full $18 \mathrm{~S}$ sequence from Acanthamoeba strains (the picture of contamination in

4 chromatograms from a clonal culture) and rarely reported cases when different $18 \mathrm{~S}$ variants

5 were obtained from the same lens of a patient (Booton et al., 2002); $\mathrm{T}_{\mathrm{A}}$ and $\mathrm{T} 4_{\mathrm{C}}$ ) or from the

6 right and left lens cases (Ledee et al., 2009); both T4в).

\section{Cox 1}

8 The strains for which Cox 1 sequences are available mostly have no full $18 \mathrm{~S}$ sequences in the

9 public domain. Our Cox 1 phylogeny fully corroborated the $16 \mathrm{~S}$ phylogeny in the proximity

10 of $18 \mathrm{~S}-\mathrm{T} 4_{\mathrm{A} / \mathrm{B}}$, as well as $18 \mathrm{~S}-\mathrm{T} 4_{\mathrm{C} / \mathrm{F}}$, strains, the distinctiveness of other subtypes, and the

11 internal heterogeneity of $\mathrm{T} 4_{\mathrm{A} / \mathrm{B}}$ clade. Here again, strain SCL-14-12 was firmly placed into

12 different cluster than all other permafrost strains, which suggests the correspondence of the

13 Cox 1 clusters to the $16 \mathrm{~S}$ ones.

\section{Morphometrics}

15 Traditional taxonomy of Acanthamoeba has been primarily based on metric (overall

16 diameter, nucleus diameter, nucleolus diameter) and meristic (number of "angles", or

17 "corners", or "arms", i.e. protrusions of endocyst towards operculi) characters of cysts since

18 Page's redescription of A. castellanii, A. polyphaga, and Acanthamoeba astronyxis (Page,

19 1967). Pussard and Pons emphasized the number of angles and properties of endo- and

20 ectocyst (smoothness, distinctiveness), which they have studied in a considerable amount of

21 strains using different staining techniques (Pussard and Pons, 1977). However, with the

22 number of strains growing, the variability in these characters also grew and has become

23 overlapping. Also, as a rule, no statistical comparison of studied characters has been

24 conducted those times, although the number of measured individuals was usually sufficient. 
1 The use of molecular methods has shown striking similarity of some of the described species,

2 e.g., Acanthamoeba terricola Pussard, 1964 and A. castellanii Neff strain (Visvesvara and

3 Balamuth, 1975); Acanthamoeba divionensis Pussard et Pons, 1977, Acanthamoeba

4 paradivionensis Pussard et Pons, 1977, Acanthamoeba mauritaniensis Pussard et Pons, 1977,

5 and Acanthamoeba rhysodes Singh, 1952 (De Jonckheere, 1983; Liu et al., 2005). Since the

6 beginning of the DNA sequencing era, the attention of investigators has been concentrated on

7 molecular phylogeny, and little attempts have been made to correspond morphometric

8 features to molecular clusters. As a result, no integrated species concept for Acanthamoeba

9 that would include both molecular and morphological characters seems to exist by now.

10 All our strains exhibit differences in the measured morphometric characters. The

11 morphometrics of locomotor forms demonstrates the relative stability of metric characters.

12 For the width, the most variable trait, the median absolute deviation (MAD) is in the range

13 from $17.4 \%$ (SCL-14-2) to $29.7 \%$ (SCL-am8) of the median (standard deviation (SD) from

$1416.5 \%$ to $25.5 \%$ ). The MAD of the length is between 9.9\% (SCL-14-3 and SCL-14-9) and

$1516.5 \%$ (SCL-am9) of the median (SD 12.1\% to 19.4\%). The level of variability found in the

16 measured characters of cysts is even lower (not surprisingly). With the number of measured

17 cells around 50, this level of variability allow to register significant differences (at $p \leq .05$ )

18 even between genetically closely related strains (Tab. 2, 3).

19 It should be stressed that the culture conditions among the strains during the taking of

20 measurement were held essentially the same to assure that the observed differences may be

21 attributed to genetics. Unsurprisingly, we have found significant variability associated with

22 the type and density of the culture in cysts (Tab. 3, Fig. 7). This indicates that the obtained

23 results are valid for the comparison of the studied strains but may not be used to compare

24 with literature data unless the conditions of culturing coincide exactly. 
1 The classifications of the strains based upon different subsets of the morphometric data

2 demonstrates different levels of similarity with molecular classification (Fig. 9). As it was

3 discussed previously, five out of six strains are identical in mitochondrial gene sequences, so

4 the only topological feature shared by all molecular trees is the most distant position of SCL-

5 14-12. Not all morphological trees share this feature as well. Specifically, this is the case for

6 locomotor form and sparse axenic cyst measurements (Fig. 9F, G), which also drives the

7 overall configuration (Fig. 9D). The dense axenic cyst and bacterized cyst data (Fig. 9H, I),

8 as well as the pseudocyst data, do not show this topology. Notably, monoclonal bacterized

9 culture on an agar plate is the most common setting in which measurements have been taken

10 from different Acanthamoeba strains, partially because not in every case a culture has been

11 axenized, and partially because this has been (probably rightly) believed to most closely

12 resemble the natural conditions. Our results suggest that measurements taken in axenic

13 conditions may better reflect genetic differences.

14 Thus, the most distant position of strain SCL-14-12 is not absolutely stable when different

15 sets of features and different culture conditions are compared. At the same time, we tend to

16 attach greater importance to the measurements of locomotor forms as to embracing more

17 different cell features. In addition to significant differences in linear dimensions, strain SCL-

18 14-12 also differs from others by its proportions, specifically the width to length, nucleolus to

19 nucleus diameter, and hyaloplasm width to cell length ratios. It should be noted that not only

20 SCL-14-12 is the most distant strain in the locomotor form-based classification, but also the

21 further branching (SCL-am9, then SCL-14-9) partially follows the order of the 18S tree (Fig.

22 9C).

23 So, to reliably compare morphometric data between strains in Acanthamoeba (and other

24 amoebae), the establishment of standardized culture conditions is absolutely necessary.

25 Axenic culture in a defined medium is the best candidate for such a standard, but the 
1 temperature should obviously be considered as well.

\section{Growth at different temperatures}

3 As with the morphometric data, we observe differences in the culture growth are between all

4 studied strains and temperatures. Generally, the higher the temperature, the higher the logistic

5 growth rate, and the lower the culture density asymptote. Also, at temperatures from $25^{\circ} \mathrm{C}$

6 and higher, multinucleate cells appear, prevailing over the normal mononucleate cells at $32^{\circ} \mathrm{C}$

7 in most strains. James and Byers (1967) also observed multinucleates in aging cultures; their

8 amount was increased by agitation or air bubbling. Corsaro et al. (2015) observed

9 multinucleates in a culture of Acanthamoeba micheli Corsaro, Walochnik, Köhsler et Rott,

10 2015. Most likely, multinucleate cells result from incomplete cell division (karyokinesis not

11 followed by cytokinesis), but the possibility of cell fusion also cannot be ruled out (Pigon,

12 1972). Our data strongly supports the first way.

13 In the growth data, strain SCL-14-12 is also obviously different from others. At $25^{\circ} \mathrm{C}$, it

14 shows approximately the same growth rate as at $18^{\circ} \mathrm{C}$, while other strains increase it. At

$1532^{\circ} \mathrm{C}$, no multinucleate cells appear in this strain, and the cells are attached to the substratum.

16 Also, the growth rate is the highest. Thus, in addition to higher growth limits at all

17 temperatures tested (a feature stemming probably from its smaller size), SCL-14-12 is

18 characterized by a broader range of temperatures suitable for normal culture growth.

19 The potential pathogenicity of Acanthamoeba strains is often addressed by testing their

20 possibility to grow at $34^{\circ} \mathrm{C}$, a temperature of the human cornea (Walochnik et al., 2000), and

$2137^{\circ} \mathrm{C}$. All permafrost strains except SCL-14-12 demonstrate clear signs of dysfunction

22 already at $32^{\circ} \mathrm{C}$, and may thus be considered non-pathogenic. For SCL-14-12, the potential to

23 cause keratitis cannot be excluded. 


\section{Conclusion}

2 Although a complex comparative study of strains inside A. castellanii complex (ribotype T4)

3 remains a future perspective, our investigation demonstrates that the results of consistently

4 applied morphometrics and ecophysiological studies (characteristics of growth at different

5 temperatures) are in line with molecular variability of several kinds of genes (nuclear and

6 mitochondrial) and may thus form a basis for Acanthamoeba-specific species concept. Amid

7 the diversity of the entire genus, all studied strains are closely related to each other. However,

8 strain SCL-14-12 is unique in all three sequenced genes, while the others possess the same

9 sequences of the mitochondrial genes. Phylogenies built with all available sequences firmly

10 place SCL-14-12 apart from the other strains. At the same time, it differs by many

11 morphometric characters of both trophozoites and cysts, including proportions, as well as

12 growth characteristics at different temperatures. Besides, directly observable features of

13 cultures also distinguish this strain from the others. Other strains also exhibit some variation

14 (and are obviously different in their 18S sequences), but this variation does not involve all

15 studied features and is smaller in magnitude. Taking all this into account, we consider strain

16 SCL-14-12 and other permafrost strains as two separate species. This conclusion may also be

17 extrapolated onto other clusters obtained in mitochondrial phylogenies inside $\mathrm{T} 4_{\mathrm{A} / \mathrm{B}}$ clade, if

18 only morphological and ecophysiological differences of strains between those clusters are

19 consistent, which needs further investigation. Until such investigation placing phenotypic

20 divergence in the framework of steady multigene phylogeny is done, a revision of $A$.

21 castellanii species complex and thus the genus as a whole seems not possible.

22 Although most of Acanthamoeba 18S rDNA types are conceivably real taxa, in A. castellanii

23 complex, the phylogenetic signal of this gene is biased, and thus it should not be used for

24 species delimitation in this group. Mitochondrial gene phylogenies seem to be more suitable 
1 for this purpose.

\section{Acknowledgements}

3 This study partially utilized microscopy equipment of the Core Facility Centres

4 "Development of molecular and cell technologies" and "Culture Collection of

5 Microorganisms" of Saint Petersburg State University (SPSU). We thank Dr. Alexei Smirnov

6 for providing the possibility to use these facilities. We are obliged to all Soil Cryology lab

7 members who participated in field sampling. We would also like to thank Prof. Dr. Jean-

8 Michel Claverie, Laboratoire Information Génomique et Structurale, Aix Marseille

9 Université, for his valuable comments on the manuscript.

10 This study was conducted in the framework of the Governmental Program 0191-2019-0044

11 and supported by Russian Foundation for Basic Research (RFBR) grants 18-04-00824

12 (isolation and culture establishment) and 17-54-150003 (description of the strains).

\section{References}

14 Amaral-Zettler, L.A., Cole, J., Laatsch, A.D., Nerad, T.A., Anderson, O.R., Reysenbach, A.L., 2006. Vannella epipetala n. sp. isolated from the leaf surface of Spondias mombin (Anacardiaceae) growing in the dry forest of Costa Rica. J. Eukaryot. Microbiol. 53,

18 Boenigk, J., Ereshefsky, M., Hoef-Emden, K., Mallet, J., Bass, D., 2012. Concepts in protistology: Species definitions and boundaries. Eur. J. Protistol. 48, 96-102. https://doi.org/10.1016/j.ejop.2011.11.004

21 Booton, G.C., Joslin, C.E., Shoff, M., Tu, E.Y., Kelly, D.J., Fuerst, P.A., 2009. Genotypic identification of Acanthamoeba sp. isolates associated with an outbreak of 
Acanthamoeba keratitis (AK). Cornea 28, 673-676. https://doi.org/10.1097/ICO.0b013e31819342a7

3 Booton, G.C., Kelly, D.J., Chu, Y.-W., Seal, D.V., Houang, E., Lam, D.S.C., Byers, T.J., Fuerst, P.A., 2002. 18S ribosomal DNA typing and tracking of Acanthamoeba species 1621-1625. https://doi.org/10.1128/JCM.40.5.1621-1625.2002 isolates from corneal scrape specimens, contact lenses, lens cases, and home water supplies of Acanthamoeba keratitis patients in Hong Kong. J. Clin. Microbiol. 40,

Byers, T.J., Bogler, S.A., Burianek, L.L., 1983. Analysis of mitochondrial DNA variation as an approach to systematic relationships in the genus Acanthamoeba. J. Protozool. 30,

14 Chung, D.I., Yu, H.S., Hwang, M.Y., Kim, T.H., Kim, T.O., Yun, H.C., Kong, H.H., 1998. Subgenus classification of Acanthamoeba by riboprinting. Korean J. Parasitol. 36, 69-

17 Clarke, M., Lohan, A.J., Liu, B., Lagkouvardos, I., Roy, S., Zafar, N., Bertelli, C., Schilde, C., Kianianmomeni, A., Bürglin, T.R., others, 2013. Genome of Acanthamoeba castellanii highlights extensive lateral gene transfer and early evolution of tyrosine kinase signaling. Genome Biol. 14, R11. https://doi.org/10.1186/gb-2013-14-2-r11 80. http://dx.doi.org/10.3347/kjp.1998.36.2.69

.

(1)

(1)
198-203. https://doi.org/10.1111/j.1550-7408.1983.tb02903.x

1 Corsaro, D., Köhsler, M., Montalbano Di Filippo, M., Venditti, D., Monno, R., Di Cave, D., Berrilli, F., Walochnik, J., 2017. Update on Acanthamoeba jacobsi genotype T15, 3 including full-length 18 S rDNA molecular phylogeny. Parasitol. Res. 116, 12731284. https://doi.org/10.1007/s00436-017-5406-1

25 Corsaro, D., Venditti, D., 2018. An apparent Acanthamoeba genotype is the product of a 
chimeric 18S rDNA artifact. Parasitol. Res. 117, 571-577. https://doi.org/10.1007/s00436-017-5690-9

3 Corsaro, D., Walochnik, J., Köhsler, M., Rott, M.B., 2015. Acanthamoeba misidentification and multiple labels: Redefining genotypes T16, T19, and T20 and proposal for Acanthamoeba micheli sp. nov. (genotype T19). Parasitol. Res. 114, 2481-2490. https://doi.org/10.1007/s00436-015-4445-8

7 De Jonckheere, J.F., 1983. Isoenzyme and total protein analysis by agarose isoelectric focusing, and taxonomy of the genus Acanthamoeba. J. Protozool. 30, 701-706.

10 Demidov, N.E., Baranskaya, A.V., Durdenko, E.V., Zanina, O.G., Karaevskaya, E.S., https://doi.org/10.1111/j.1550-7408.1983.tb05346.x

14 Elzhov, T.V., Mullen, K.M., Spiess, A.-N., Bolker, B., 2016. Minpack.lm: R interface to the Pushina, Z.V., Rivkina, E.M., Spirina, E.V., Spenser, R., 2016. [Biogeochemistry of

18 Fuerst, P.A., 2014. Insights from the DNA databases: Approaches to the phylogenetic structure of Acanthamoeba. Exp. Parasitol. 145, S39-S45.

21 Fuerst, P.A., Booton, G.C., Crary, M., 2015. Phylogenetic analysis and the evolution of the 18S rRNA gene typing system of Acanthamoeba. J. Eukaryot. Microbiol. 62, 69-84.

24 Gast, R.J., Ledee, D.R., Fuerst, P.A., Byers, T.J., 1996. Subgenus systematics of Acanthamoeba: Four nuclear 18S rDNA sequence types. J. Eukaryot. Microbiol. 43, 
498-504. https://doi.org/10.1111/j.1550-7408.1996.tb04510.x

2 Gast, R.J., Moran, D.M., Dennett, M.R., Wurtsbaugh, W.A., Amaral- Zettler, L.A., 2011. Amoebae and Legionella pneumophila in saline environments. J. Water Health 9, 3752. https://doi.org/10.2166/wh.2010.103

5 Geisen, S., Fiore-Donno, A.M., Walochnik, J., Bonkowski, M., 2014. Acanthamoeba

8 Gilichinskiy, D.A., Khlebnikova, G.M., Zvyagintsev, D.G., Fedorov-Davydov, D.G., Kudryavtseva, N.N., 1989. Microbiology of sedimentary materials in the permafrost

11 Gilichinsky, D.A., Rivkina, E.M., 2011. Permafrost microbiology, in: Reitner, J., Thiel, V. (Eds.), Encyclopedia of Geobiology, Encyclopedia of Earth Sciences Series. Springer

14 Gong, J., Dong, J., Liu, X., Massana, R., 2013. Extremely high copy numbers and https://doi.org/10.1016/j.protis.2012.11.006

18 Gribble, K.E., Anderson, D.M., 2007. High intraindividual, intraspecific, and interspecific variability in large-subunit ribosomal DNA in the heterotrophic dinoflagellates Protoperidinium, Diplopsalis, and Preperidinium (Dinophyceae). Phycologia 46,

22 Gunderson, J.H., Sogin, M.L., 1986. Length variation in eukaryotic rRNAs: Small subunit rRNAs from the protists Acanthamoeba castellanii and Euglena gracilis. Gene 44, 63-70. https://doi.org/10.1016/0378-1119(86)90043-0

25 James, T.E., Byers, T.J., 1967. The induction of multinuclearity in agitated and in aging 
cultures of Acanthamoeba sp. Neff. J. Cell. Physiol. 70, 53-61. https://doi.org/10.1002/jcp.1040700108

3 Juck, D.F., Whissell, G., Steven, B., Pollard, W., McKay, C.P., Greer, C.W., Whyte, L.G., 2005. Utilization of fluorescent microspheres and a green fluorescent protein-marked strain for assessment of microbiological contamination of permafrost and ground ice core samples from the Canadian High Arctic. Appl. Environ. Microbiol. 71, 10351041. https://doi.org/10.1128/AEM.71.2.1035-1041.2005

Katoh, K., Standley, D.M., 2013. MAFFT multiple sequence alignment software version 7: improvements in performance and usability. Mol. Biol. Evol. 30, 772-780. https://doi.org/10.1093/molbev/mst010

13 Kliescikova, J., Kulda, J., Nohynkova, E., 2011. Stress-induced pseudocyst formation - a newly identified mechanism of protection against organic solvents in acanthamoebae of the T4 genotype. Protist 162, 58-69. https://doi.org/10.1016/j.protis.2010.03.006

Khan, N.A., 2005. Granulomatous amoebic encephalitis: Clinical diagnosis and management. Am. J. Infect. Dis. 1, 79-83. https://doi.org/10.3844/ajidsp.2005.79.83

Kong, H.H., 2009. Molecular phylogeny of Acanthamoeba. Korean J. Parasitol. 47, S21-S28. https://doi.org/10.3347/kjp.2009.47.S.S21

22 Kudryavtsev, A., Gladkikh, A., 2017. Two new species of Ripella (Amoebozoa, Vannellida) and unusual intragenomic variability in the SSU rRNA gene of this genus. Eur. J. Protistol. 61, 92-106. https://doi.org/10.1016/j.ejop.2017.09.003

25 Ledee, D.R., Booton, G.C., Awwad, M.H., Sharma, S., Aggarwal, R.K., Niszl, I.A., Markus, 

sequences to classify clinical isolates of Acanthamoeba. Investig. Opthalmology Vis. Sci. 44, 1142. https://doi.org/10.1167/iovs.02-0485 2009. Molecular identification of T4 and T5 genotypes in isolates from Acanthamoeba keratitis patients. J. Clin. Microbiol. 47, 1458-1462.

Legendre, M., Bartoli, J., Shmakova, L., Jeudy, S., Labadie, K., Adrait, A., Lescot, M., Poirot, O., Bertaux, L., Bruley, C., others, 2014. Thirty-thousand-year-old distant relative of giant icosahedral DNA viruses with a pandoravirus morphology. Proc. Natl. Acad. Sci. 111, 4274-4279. https://doi.org/10.1073/pnas.1320670111

Liu, H., Ha, Y.-R., Lee, S.-T., Hong, Y.-C., Kong, H.-H., Chung, D.-I., 2006. Genetic diversity of Acanthamoeba isolates from ocean sediments. Korean J. Parasitol. 44, 117. https://dx.doi.org/10.3347/kjp.2006.44.2.117

Liu, H., Moon, E.-K., Yu, H.-S., Jeong, H.-J., Hong, Y.-C., Kong, H.-H., Chung, D.-I., 2005. Evaluation of taxonomic validity of four species of Acanthamoeba: A. divionensis, A. paradivionensis, A. mauritaniensis, and A. rhysodes, inferred from molecular analyses. Korean J. Parasitol. 43, 7-13. https://doi.org/10.3347/kjp.2005.43.1.7

Maciver, S.K., Asif, M., Simmen, M.W., Lorenzo-Morales, J., 2013. A systematic analysis of Acanthamoeba genotype frequency correlated with source and pathogenicity: T4 is confirmed as a pathogen-rich genotype. Eur. J. Protistol. 49, 217-221. https://doi.org/10.1016/j.ejop.2012.11.004

Martín-Pérez, T., Criado-Fornelio, A., Martínez, J., Blanco, M.A., Fuentes, I., Pérez-Serrano, J., 2017. Isolation and molecular characterization of Acanthamoeba from patients with keratitis in Spain. Eur. J. Protistol. 61, 244-252. 
https://doi.org/10.1016/j.ejop.2017.06.009

2 Maycock, N.J.R., Jayaswal, R., 2016. Update on Acanthamoeba keratitis: diagnosis, treatment, and outcomes. Cornea 35, 713. https://doi.org/10.1097/ICO.0000000000000804

5 Megha, K., Sharma, M., Gupta, A., Sehgal, R., Khurana, S., 2018. Protein profiling of Acanthamoeba species using MALDI-TOF MS for specific identification of Acanthamoeba genotype. Parasitol. Res. 117, 729-736.

9 Nassonova, E., Smirnov, A., Fahrni, J., Pawlowski, J., 2010. Barcoding amoebae: comparison of SSU, ITS and COI genes as tools for molecular identification of naked lobose amoebae. Protist 161, 102-115. https://doi.org/10.1016/j.protis.2009.07.003 https://doi.org/10.1007/s00436-017-5743-0

15 Nguyen, L.-T., Schmidt, H.A., von Haeseler, A., Minh, B.Q., 2015. IQ-TREE: A fast and effective stochastic algorithm for estimating maximum-likelihood phylogenies. Mol.

18 Ntakou, E., Siemensma, F., Bonkowski, M., Dumack, K., 2019. The dancing star: reinvestigation of Artodiscus saltans (Variosea, Amoebozoa) Penard 1890. Protist 170, 349-357. https://doi.org/10.1016/j.protis.2019.06.002 Biol. Evol. 32, 268-274. https://doi.org/10.1093/molbev/msu300

Nuprasert, W., Putaporntip, C., Pariyakanok, L., Jongwutiwes, S., 2010. Identification of a novel T17 genotype of Acanthamoeba from environmental isolates and T10 genotype causing keratitis in Thailand. J. Clin. Microbiol. 48, 4636-4640. https://doi.org/10.1128/JCM.01090-10

5 Oksanen, J., Blanchet, F.G., Friendly, M., Kindt, R., Legendre, P., McGlinn, D., Minchin, 

Wagner, H., 2019. Vegan: community ecology package. URL https://CRAN.Rproject.org/package=vegan (accessed 7.8.19).

4 Page, F.C., 1988. A new key to freshwater and soil Gymnamoebae: with instructions for culture. Freshwater Biol. Assoc., Ambleside.

6 Page, F.C., 1967. Re-definition of the genus Acanthamoeba with descriptions of three species. J. Protozool. 14, 709-724. https://doi.org/10.1111/j.1550-

9 Pigon, A., 1972. Inhibition of movement, attachment, and cytokinesis by autogenous substances in the amoeba Hartmannella. Exp. Cell Res. 73, 170-176. https://doi.org/10.1016/0014-4827(72)90115-2

12 Pillet, L., Fontaine, D., Pawlowski, J., 2012. Intra-genomic ribosomal RNA polymorphism and morphological variation in Elphidium macellum suggests inter-specific

16 Pussard, M., Pons, R., 1977. Morphologie de la paroi kystique et taxonomie du genre Acanthamoeba (Protozoa, Amoebida). Protistologica 13, 557-598.

18 Qvarnstrom, Y., Nerad, T.A., Visvesvara, G.S., 2013. Characterization of a new pathogenic Acanthamoeba species, A. byersi n. sp., isolated from a human with fatal amoebic encephalitis. J. Eukaryot. Microbiol. 60, 626-633. https://doi.org/10.1111/jeu.12069

21 R Core Team, 2019. R: a language and environment for statistical computing. R Foundation for Statistical Computing, Vienna, Austria. URL https://www.R-project.org/ (accessed 7.8.19).

24 Rahman, M.M., Yagita, K., Kobayashi, A., Oikawa, Y., Hussein, A.I.A., Matsumura, T., 
Japan using nuclear and mitochondrial small subunit ribosomal RNA. Korean J.

3 Rivkina, E.M., Friedmann, E.I., McKay, C.P., Gilichinsky, D.A., 2000. Metabolic activity of permafrost bacteria below the freezing point. Appl. Environ. Microbiol. 66, 32303233. https://doi.org/10.1128/AEM.66.8.3230-3233.2000

6 Ronquist, F., Teslenko, M., van der Mark, P., Ayres, D.L., Darling, A., Höhna, S., Larget, B., Liu, L., Suchard, M.A., Huelsenbeck, J.P., 2012. MrBayes 3.2: Efficient Bayesian phylogenetic inference and model choice across a large model space. Syst Biol 61, 539-542. https://doi.org/10.1093/sysbio/sys029

10 Schirrmeister, L., Kunitsky, V., Grosse, G., Wetterich, S., Meyer, H., Schwamborn, G., Babiy, O., Derevyagin, A., Siegert, C., 2011. Sedimentary characteristics and origin

Shatilovich, A., Stoupin, D., Rivkina, E., 2015. Ciliates from ancient permafrost: Assessment of cold resistance of the resting cysts. Eur. J. Protistol. 51, 230-240. https://doi.org/10.1016/j.ejop.2015.04.001

Shatilovich, A.V., Shmakova, L.A., Mylnikov, A.P., Gilichinsky, D.A., 2009. Ancient Protozoa isolated from permafrost, in: Margesin, R. (Ed.), Permafrost Soils. Springer Berlin Heidelberg, Berlin, Heidelberg, pp. 97-115. https://doi.org/10.1007/978-3-54069371-0_8

Shatilovich, A.V., Tchesunov, A.V., Neretina, T.V., Grabarnik, I.P., Gubin, S.V., Vishnivetskaya, T.A., Onstott, T.C., Rivkina, E.M., 2018. Viable nematodes from Late Pleistocene permafrost of the Kolyma River Lowland. Dokl. Biol. Sci. 480, 100- 
2 Shi, T., Reeves, R.H., Gilichinsky, D.A., Friedmann, E.I., 1997. Characterization of viable bacteria from Siberian permafrost by $16 \mathrm{~S}$ rDNA sequencing. Microb. Ecol. 33, 169179. https://doi.org/10.1007/s002489900019

5 Shmakova, L., Bondarenko, N., Smirnov, A., 2016. Viable species of Flamella (Amoebozoa:

14 Simmons, M., Ochoterena, H., 2000. Gaps as characters in sequence-based phylogenetic

16 Stothard, D.R., Schroeder-Diedrich, J.M., Awwad, M.H., Gast, R.J., Ledee, D.R., RodriguezZaragoza, S., Dean, C.L., Fuerst, P.A., Byers, T.J., 1998. The evolutionary history of the genus Acanthamoeba and the identification of eight new 18S rRNA gene sequence types. J. Eukaryot. Microbiol. 45, 45-54.

Stoupin, D., Kiss, A.K., Arndt, H., Shatilovich, A.V., Gilichinsky, D.A., Nitsche, F., 2012. Cryptic diversity within the choanoflagellate morphospecies complex Codosiga

24 Tice, A.K., Shadwick, L.L., Fiore-Donno, A.M., Geisen, S., Kang, S., Schuler, G.A., Spiegel, F.W., Wilkinson, K.A., Bonkowski, M., Dumack, K., Lahr, D.J.G., Voelcker, E., 
Clauß, S., Zhang, J., Brown, M.W., 2016. Expansion of the molecular and morphological diversity of Acanthamoebidae (Centramoebida, Amoebozoa) and identification of a novel life cycle type within the group. Biol. Direct 11. https://doi.org/10.1186/s13062-016-0171-0

5 Vishnivetskaya, T.A., 2009. Viable cyanobacteria and green algae from the permafrost Heidelberg, pp. 73-84. https://doi.org/10.1007/978-3-540-69371-0_6

8 Visvesvara, G.S., Balamuth, W., 1975. Comparative studies on related free-living and pathogenic amebae with special reference to Acanthamoeba. J. Protozool. 22, 245-

11 Walochnik, J., Obwaller, A., Aspöck, H., 2000. Correlations between morphological,

15 Yashina, S., Gubin, S., Maksimovich, S., Yashina, A., Gakhova, E., Gilichinsky, D., 2012. molecular biological, and physiological characteristics in clinical and nonclinical isolates of Acanthamoeba spp. Appl. Environ. Microbiol. 66, 4408-4413. http://doi.org/10.1128/AEM.66.10.4408-4413.2000

19 Zanina, O.G., Gubin, S.V., Kuzmina, S.A., Maximovich, S.V., Lopatina, D.A., 2011. LatePleistocene (MIS 3-2) palaeoenvironments as recorded by sediments, palaeosols, and ground-squirrel nests at Duvanny Yar, Kolyma lowland, northeast Siberia. Quat. Sci. Rev., Beringia and Beyond: Papers Celebrating the Scientific Career of Andrei Vladimirovich Sher, 1939-2008 30, 2107-2123. https://doi.org/10.1016/j.quascirev.2011.01.021 
2 Figure 1 A-E Locomotor forms: A - SCL-14-3; B - SCL-14-3; C - SCL-14-12, D - SCLam9; E - SCL-14-3; F Floating form, SCL-am8; G - multinucleate trophozoite, SCLam9; H Monolayer, SCL-14-12; I, K-N Cysts, various strains; J Pseudocysts, SCLam8; O Empty cyst, SCL-am8.

6 Figure 2 ML (A) and BI (B) 18S SSU trees of Acanthamoeba T4 genotype showing seven subtypes. Branch supports are given as bootstrap percentage / Bayesian posterior probability. The trees are rooted at midpoint. Clades with variable position are dashed. Scale bar: 0.01 substitution per site for both A and B.

10 Figure 3 18S SSU ML tree of T4A and T4B clades (a subtree of Fig. 5A, expanded). Support values inside the clades are omitted. The tree is rooted at midpoint.

14 Figure 4 16S SSU ML tree of Acanthamoeba T4 genotype. BA tree gave the same topology. Branch supports are given as ML bootstrap percentage / Bayesian posterior probability. $\bullet-100 \%$ or 1.00 support. Support values near tree tips are omitted. The

18 Figure 5 Cox 1 ML-tree of Acanthamoeba T4 genotype rooted at midpoint. BA-tree gave the same topology. Branch supports are given as ML-bootstrap percentage / Bayesian posterior probability. $\bullet-100 \%$ or 1.00 support. * - Identification is dubious. Support values lower than $95 / .75$ are not shown.

22 Figure 6 Locomotor form length in studied strains.

23 Figure 7 Diameter of rounded cell, cyst, and pseudocyst of the studied strains in different 
conditions.

2 Figure 8 A - Scatter-plot representing modeled parameters of the logistic growth of the studied strains at different temperatures. Abscissa $-\mathrm{L}$, ordinate $-\mathrm{k}$. Lines connect points belonging to the same strain. $\mathbf{B}$ - Cluster diagram of all three logistic model parameters (scaled). Euclidean distance, Ward's clustering method.

6 Figure 9 Classification of the strains based on different data. Gene trees: A - Cox 1 NJ tree, observed distances; $\mathbf{B}-16 \mathrm{~S}$ NJ tree, observed distances; $\mathbf{C}-18 \mathrm{~S}$ tree extracted from the complete tree of T4 type. Morphometric trees: D - All features included; E Cysts and pseudocysts only; $\mathbf{F}$ - Trophozoites only; $\mathbf{G}$ - "Sparse axenic" cysts; $\mathbf{H}$ "Dense axenic" cysts; I - "Bacterized culture" cysts. All trees are rooted at midpoint. Not to scale. 

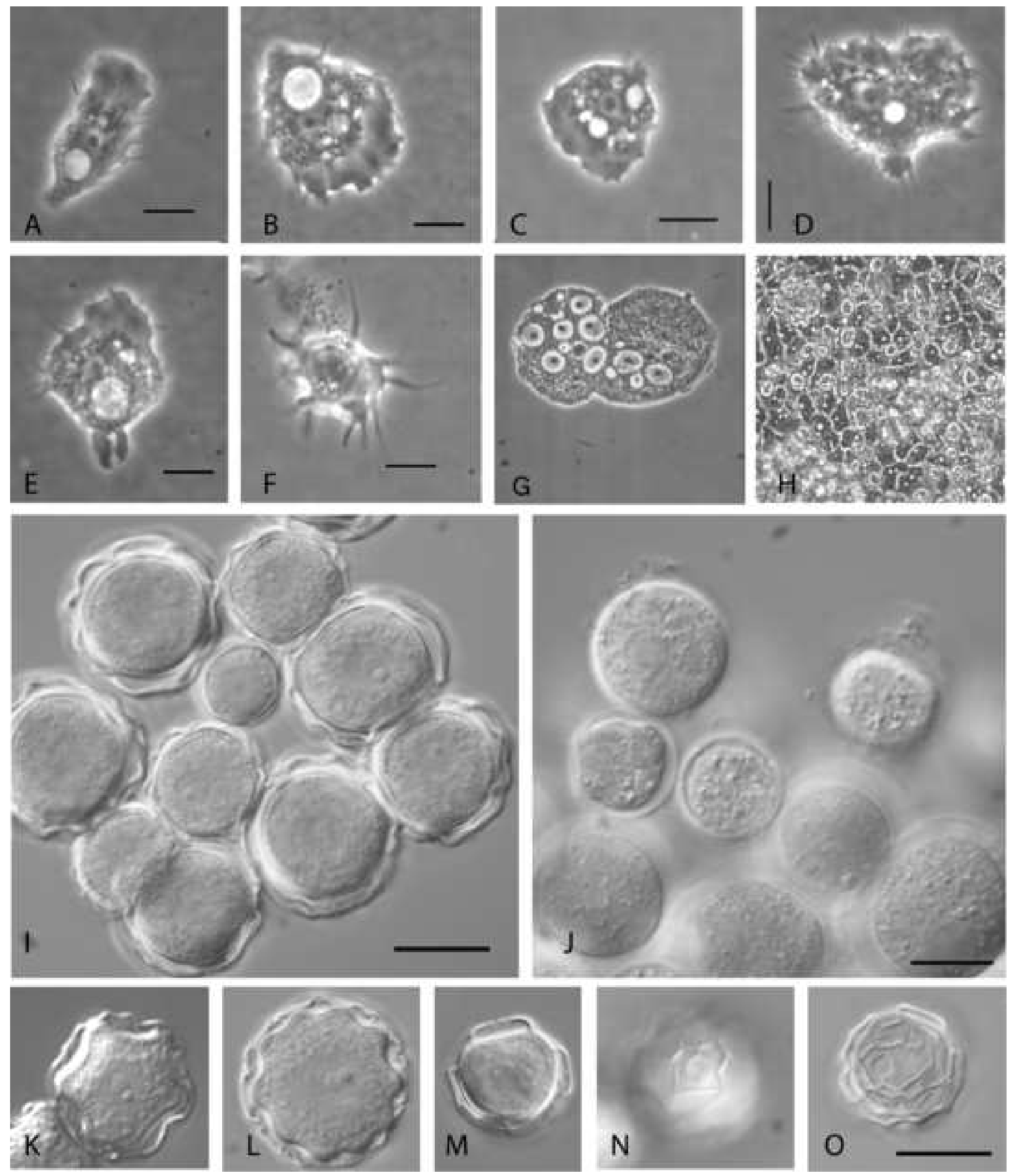


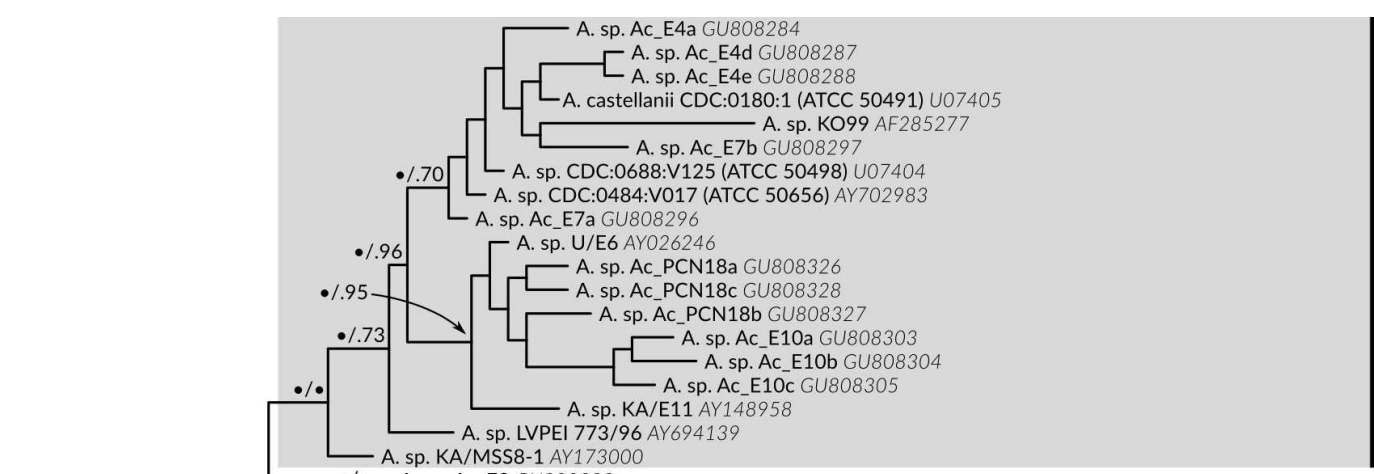

"V017" clade

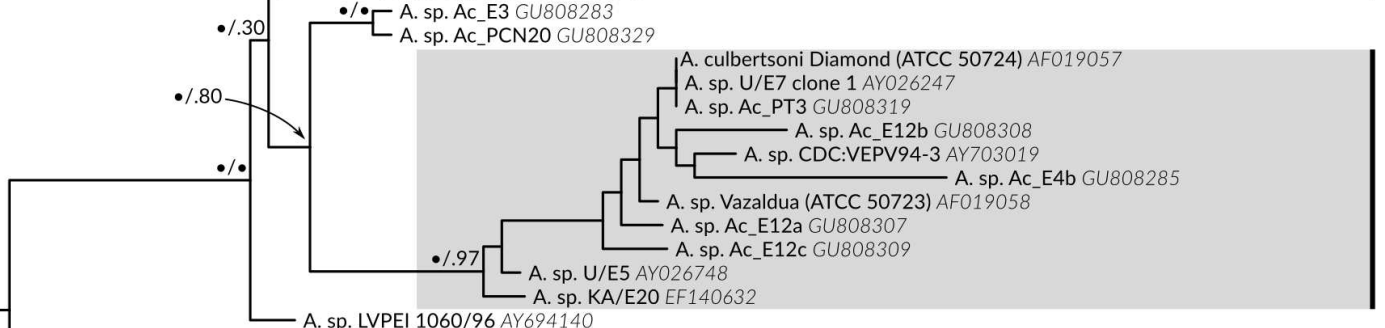

"Diamond" clade
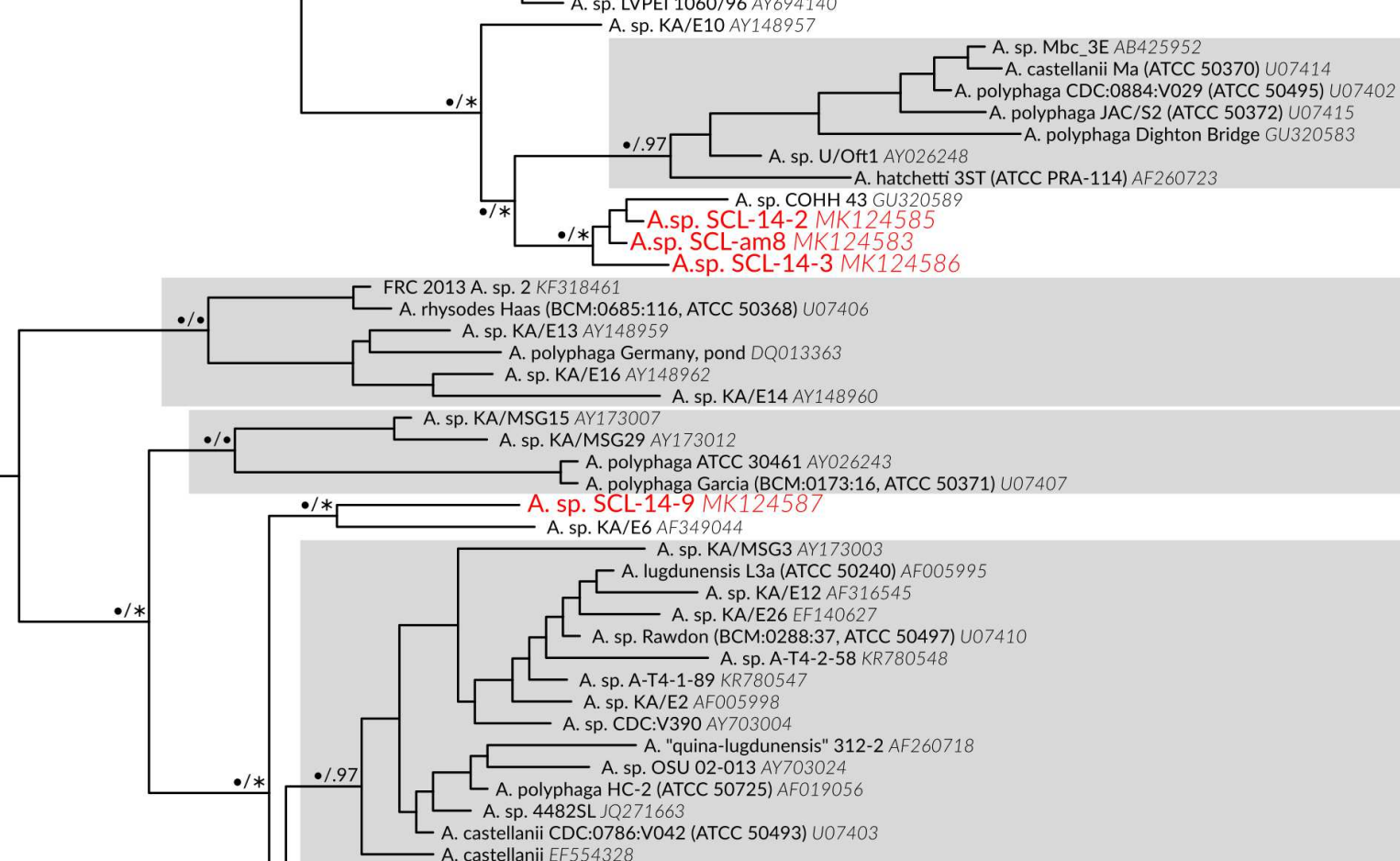

A. sp. 2 KF318461

A.sp. SCL-14-3 MK124586

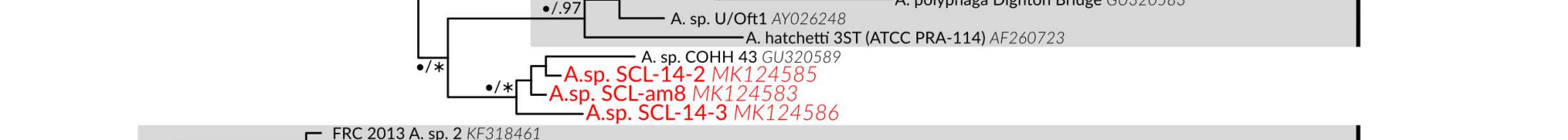

A. polyphaga Germany, pond DQ013363

A. polyphaga Germany, A. sp. KA/E14 AY148960

sp. KA/MSG15 AY173007 A. sp. KA/MSG29 AY173012

A. polyphaga ATCC 30461 AY026243

A. polyphaga Garcia (BCM:0173:16, ATCC 50371) U07407

A. sp. SCL-14-9 MK124587

A. sp. KA/E6 AF349044

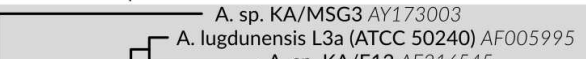

A. A. sp. KA/E12 AF31654

A. sp. Rawdon (BCM:0288:37, ATCC 50497) U07410

A. sp. A-T4-2-58 KR780548

A. sp. A-T4-1-89 KR78054

A. sp. CDC:V390 AY703004

A. "quina-lugdunensis" 312-2 AF260718

A. sp. OSU 02-013 AY703024

A. polyphaga HC-2 (ATCC 50725) AF019056

A. sp. 4482SL JQ271663

A. castellanii CDC:0786:V042 (ATCC 50493) U07403

A. castellanii EF554328
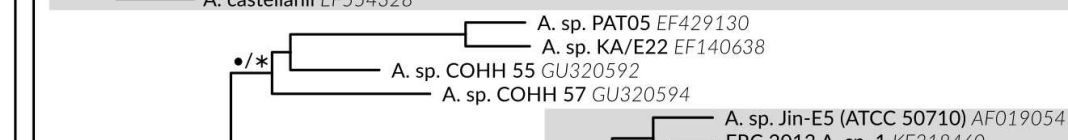

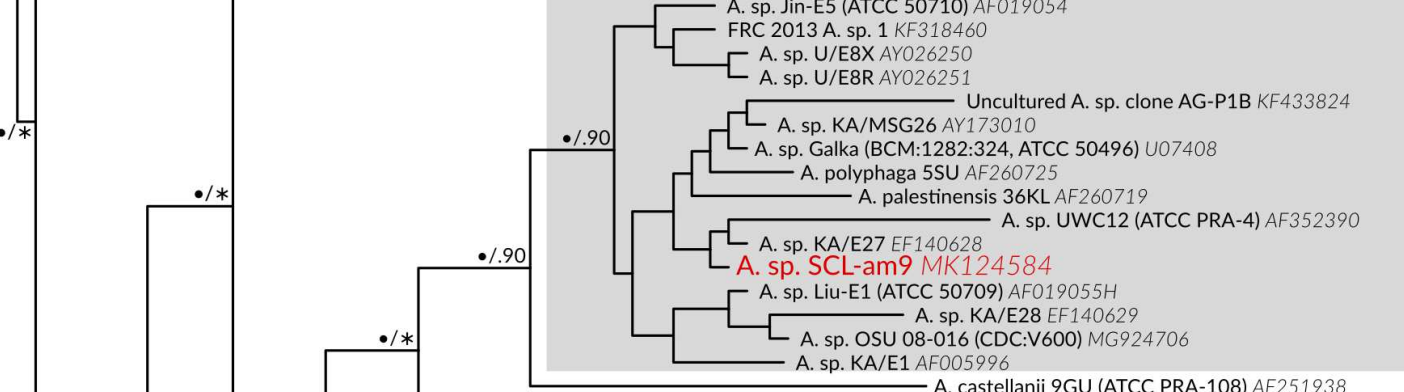

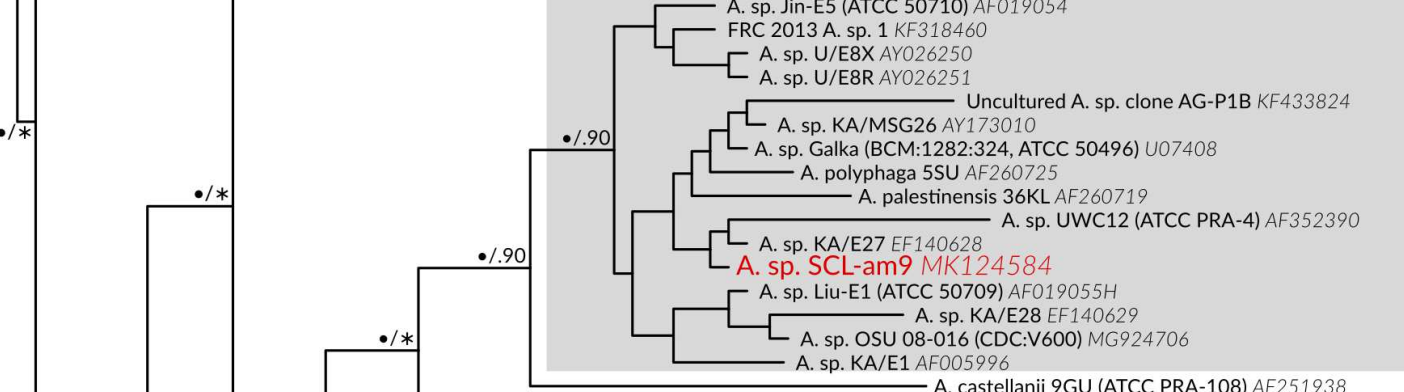

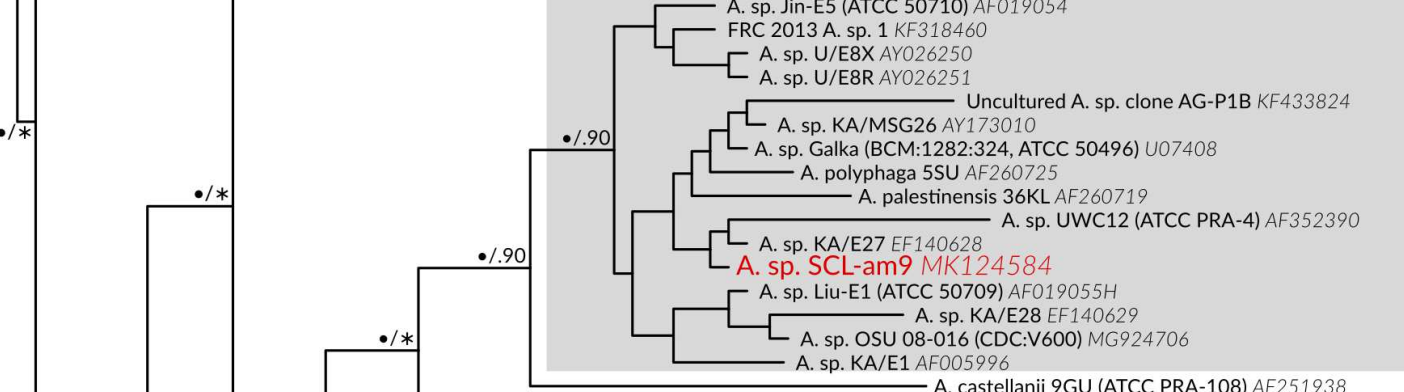

"Haas" clade

"Garcia" clade
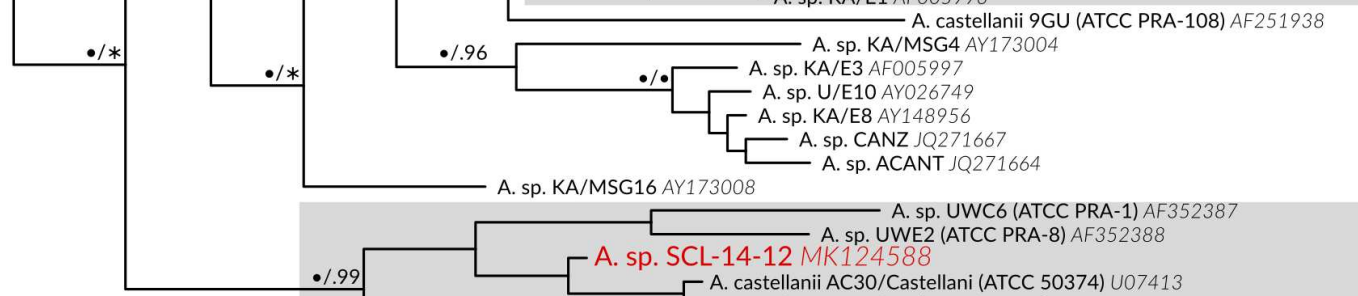

LA castellanii 4CL (ATCC PRA-107) A. castella

"Rawdon" clade

"Castellani" clade 
A. castellanii AC4 MF563606
A. sp. JPH24 AB795716

SCL-14-12 MK100248

A. sp. Jac324.jpn (Galka) AF479505

A. sp. CDC:V328 AF479501

A. castellanii JacE3 AF479498

$\because / \bullet \quad$ A. sp. CDC:V382 AF479502

A. polyphaga UNAM HC-2 AF479496

A. castellanii JacE2 AF479497

80/.98 A. sp. Galka (BCM:1282:324, ATCC 50496) AF479499

A. sp. CDC:V390 AF479503

•/• A. sp. Liu-E1 (ATCC 50709) AF479500

A. sp. OHSU M002 AF479504

A rhysodes Haas (BCM:0685:116, ATCC 50368) AF479553

A. polyphaga AC15 MF538588

A. polyphaga AC28 MF538587
A. sp. CDC:0589:V168 (ATCC 50659) AF479525

A. polyphaga AC16 MF538586

A. polyphaga AC11 MF538585

98/.99 SCL-14-2 MK100245

SCL-am9 MK100244

SCL-14-3 MK100246

SCL-14-9 MK100247

SCL-am8 MK100243

A. polyphaga AC20 MF538584

A. sp. JPH19 AB795713

A. sp. JPH14 AB795710

A. polyphaga CDC:0884:V029 (ATCC 50495) AF479526

A. sp. JPH17 AB795711

A. sp. JPH27 AB795718

A. castellanii Castellani (ATCC 50374) AF479528

72/.73 1 A. castellanii CDC:0786:V042 (ATCC 50493) AF479529

A. sp. JacKampf AF479532

A. sp. Jac473U AF479530

A. sp. JacE7 AF479531

A. castellanii Ma (ATCC 50370) AF479533

•/• A. sp. JPH18 AB795712

A. sp. JPH2 AB795705

A. sp. JPH22 AB795714

A. sp. CDC:0688:V125 (ATCC 50498) AF479524

A. culbertsoni Diamond (ATCC 50724) AF479521

A. polyphaga TV8 AF479522

76/.90 94/ A. castellanii CDC:0180:1 (ATCC 50491) AF479520

94/• A. sp. VEPV 94-3 AF479519

A. sp. CDC:0484:V017 (ATCC 50656) AF479523

[ A. sp. LVPEI 1002/99 AF479551

A. sp. LVPEI 1060/96 AF479549

$92 / .99$

A. sp. LVPEI 773/96 AF479507

•/•- A. sp. JPH5 AB795706

A. polyphaga Garcia (BCM:0173:16, ATCC 50371) AF479557

A. sp. JPH10 AB795709

-/. A. sp. Jac9E AF479556

A. sp. Rawdon (BCM:0288:37, ATCC 50497) AF479554

A. sp. CDC:VE67/H7 AF479534

A. castellanii JacE4 AF479555

A. royreba Oak Ridge (ATCC 30884) AF479559

A. rhysodes Singh (ATCC 30973) EU515177

L A. mauritaniensis 1652 (ATCC 50253) EU515180

"V168" clade

(24/.99

[A. sp. LVPEI 749/98 AF479552

A. sp. LVPEI 98/00 AF479509

•.• A. divionensis AA1 (ATCC 50251) EU515179

A. divionensis AA2 (ATCC 50238) EU515178

- A. sp. LVPEI 1035/99 AF479508

A. polyphaga Nagington (CCAP:1501/3d, ATCC 30873) AF479537

"Castellani/Ma" clade

"Diamond/V017" clade

$T 4_{A / B}$

•/. A. terricola (ATCC 30134) AF479561

A. castellanii Neff (ATCC 50373) AF479560

-/ A. castellanii AC13 MF563608

A. castellanii AC25 MF563607
A. sp. Fernandez (ATCC 50369) AF479558
A. triangularis SH621 (ATCC 50254) EU515183

A. castellanii AC25 MF563607
A. sp. Fernandez (ATCC 50369) AF479558
A. triangularis SH621 (ATCC 50254) EU515183

A. castellanii AC25 MF563607
A. sp. Fernandez (ATCC 50369) AF479558
A. triangularis SH621 (ATCC 50254) EU515183

T4

./. A. castellanii CDC:0184:V014 (ATCC 50492) AF479550

81/.97 - A. sp. KA/E23 EU515182

A. sp. LVPEI 402/97 AF479506

A. mauritaniensis AC31 MF563605

•/• A. mauritaniensis AC29 MF563604

. SAWE/SAWL sequences AF479510-AF479518

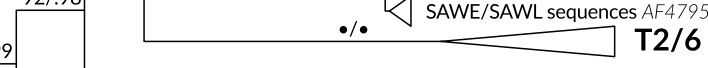


ГA. sp. OSU 05-011 MG924676

A. sp. OSU 06-051 MG924685

A. sp. OSU 08-005 MG924705

A. castellanii $C D F L$

A. sp. OSU 07-032 MG924697

A. pearcei $C D F J$ *

$99 / \bullet$

A. sp. OSU 06-005 MG924681

A. sp. OSU 07-004 MG924688

A. sp. CDC:0484:V017 (ATCC 50656) MG924712

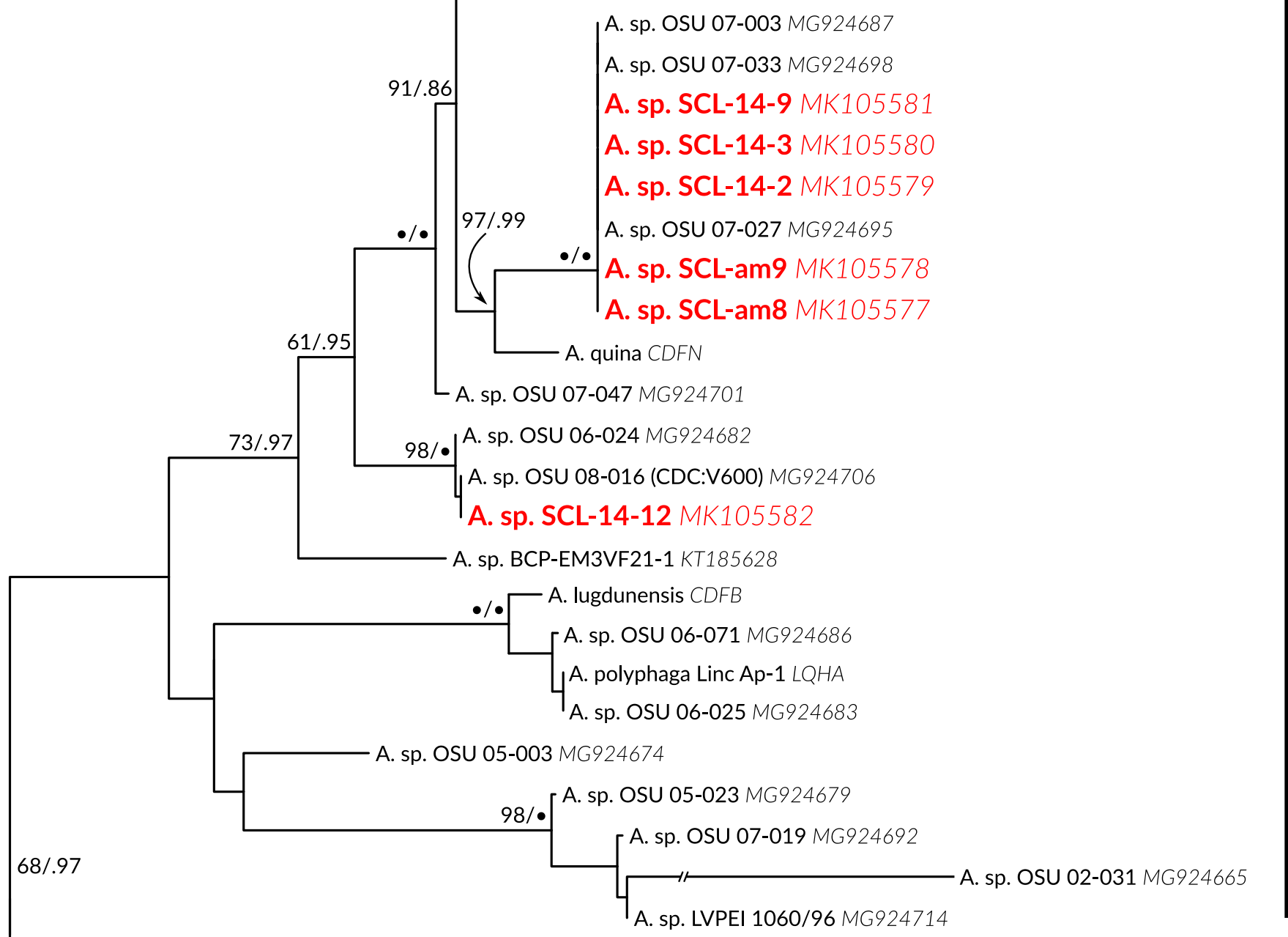

$\mathrm{T} 4 \mathrm{~A} / \mathrm{B}$
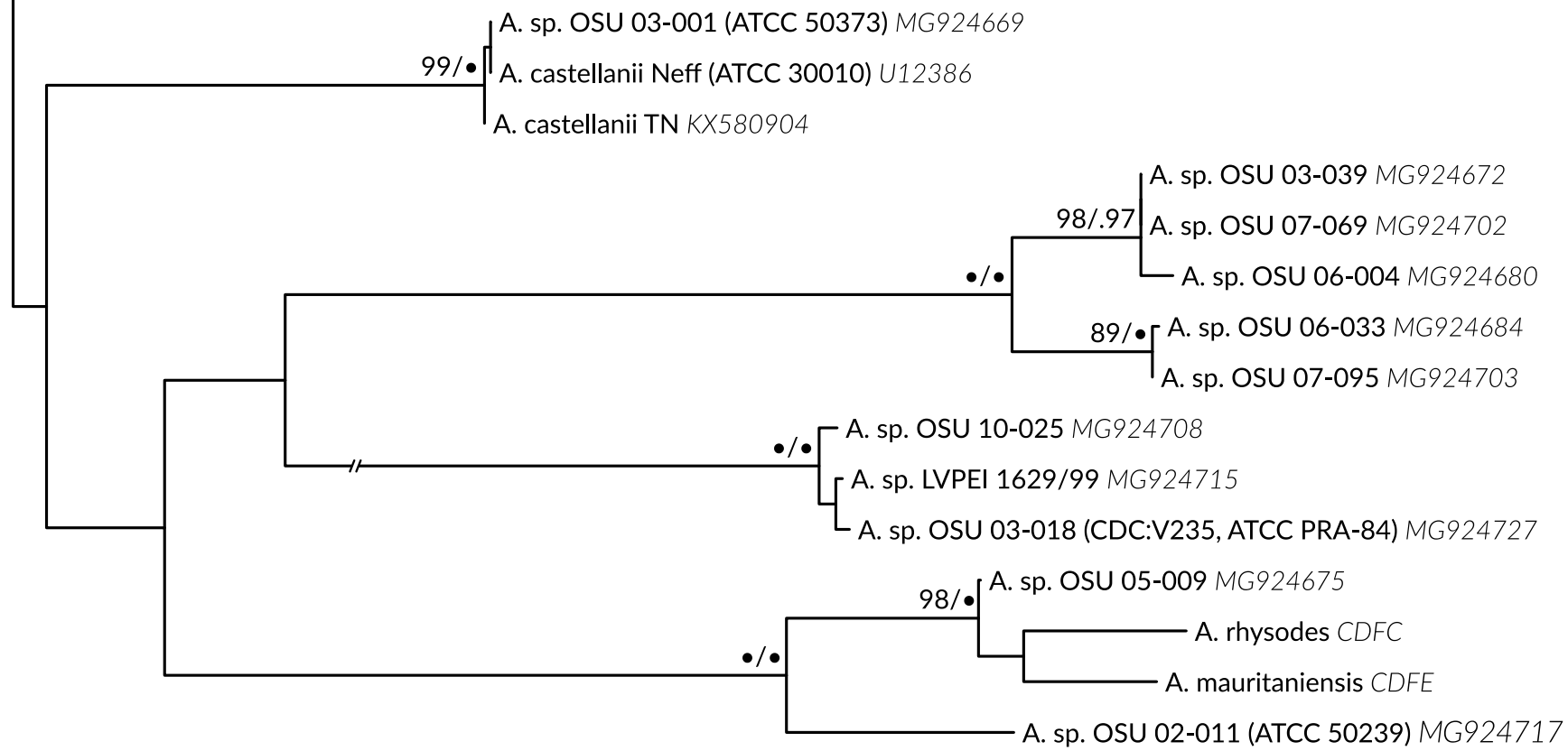

T4Neff

T4E

$\mathrm{T} 4 \mathrm{C} / \mathrm{F}$ 

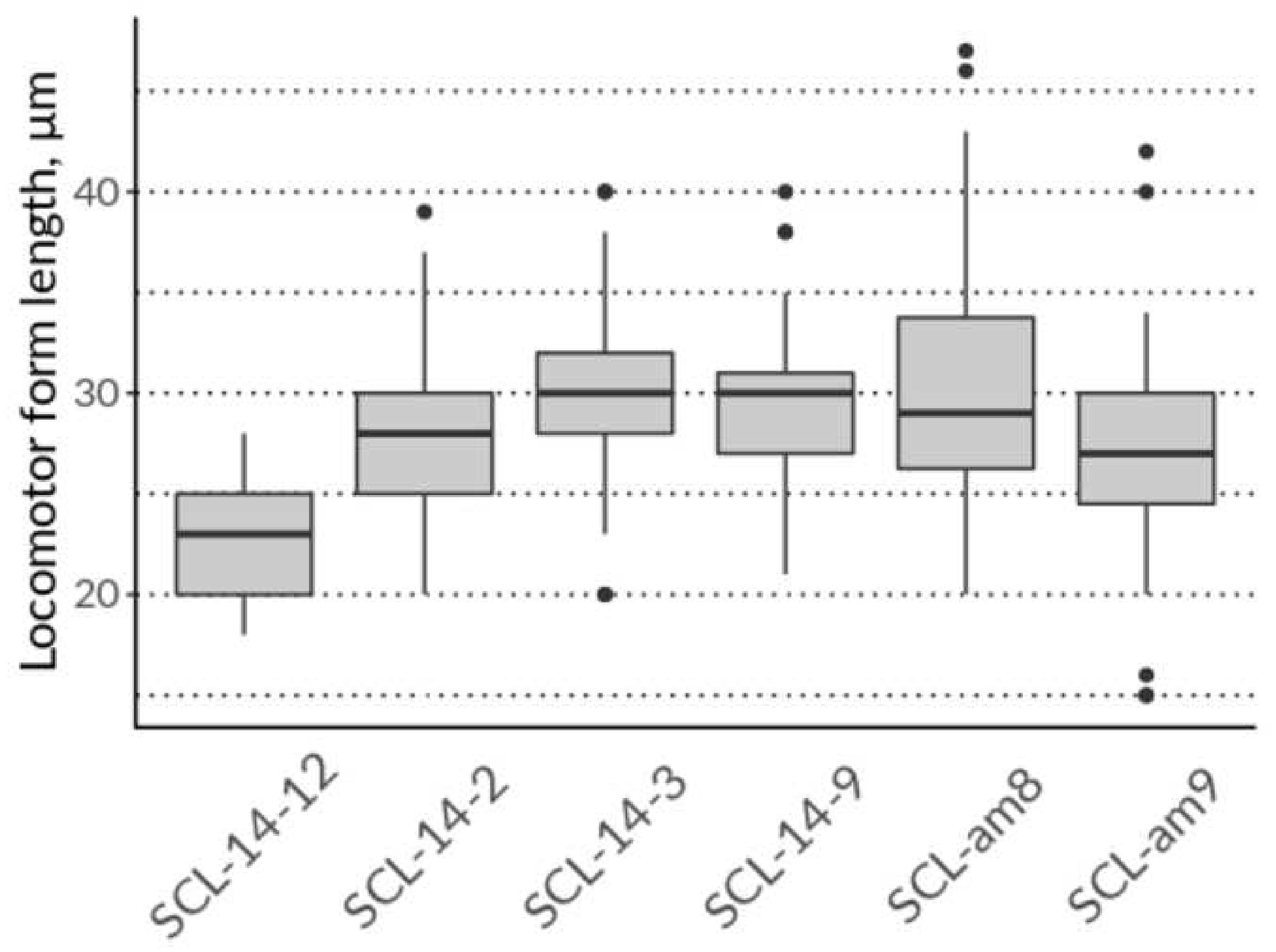


\section{Pseudocysts \\ $\square$ Cysts (bact.) \\ $\square$ Cysts (axenic-dense) \\ $\square$ Cysts (axenic-sparse) \\ Rounded troph.}

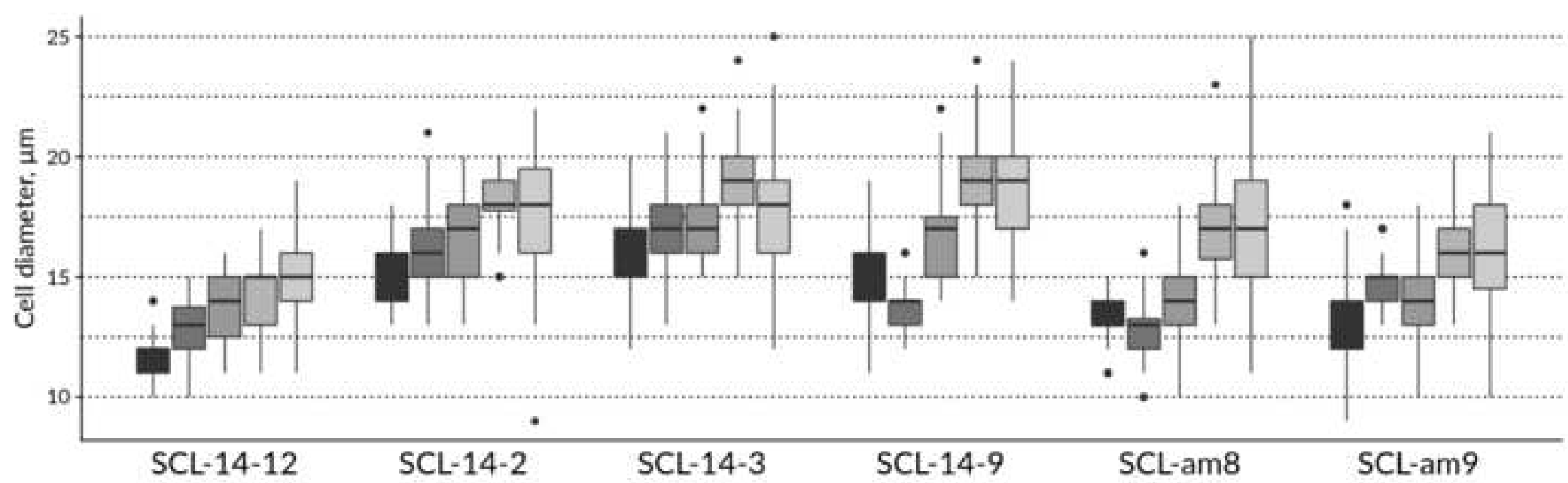


Figure 8

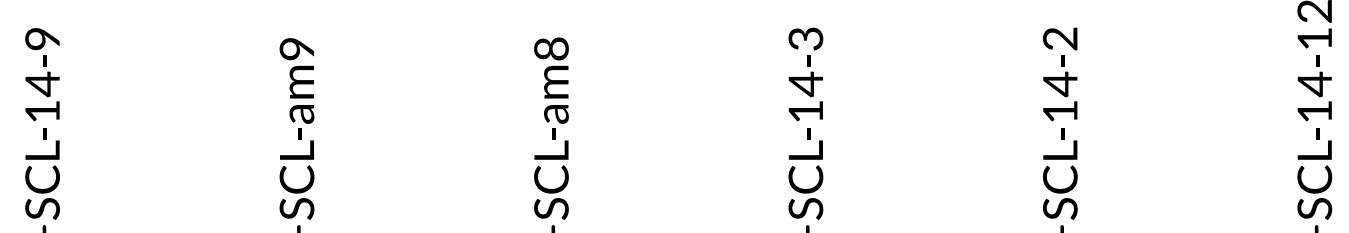

$m$

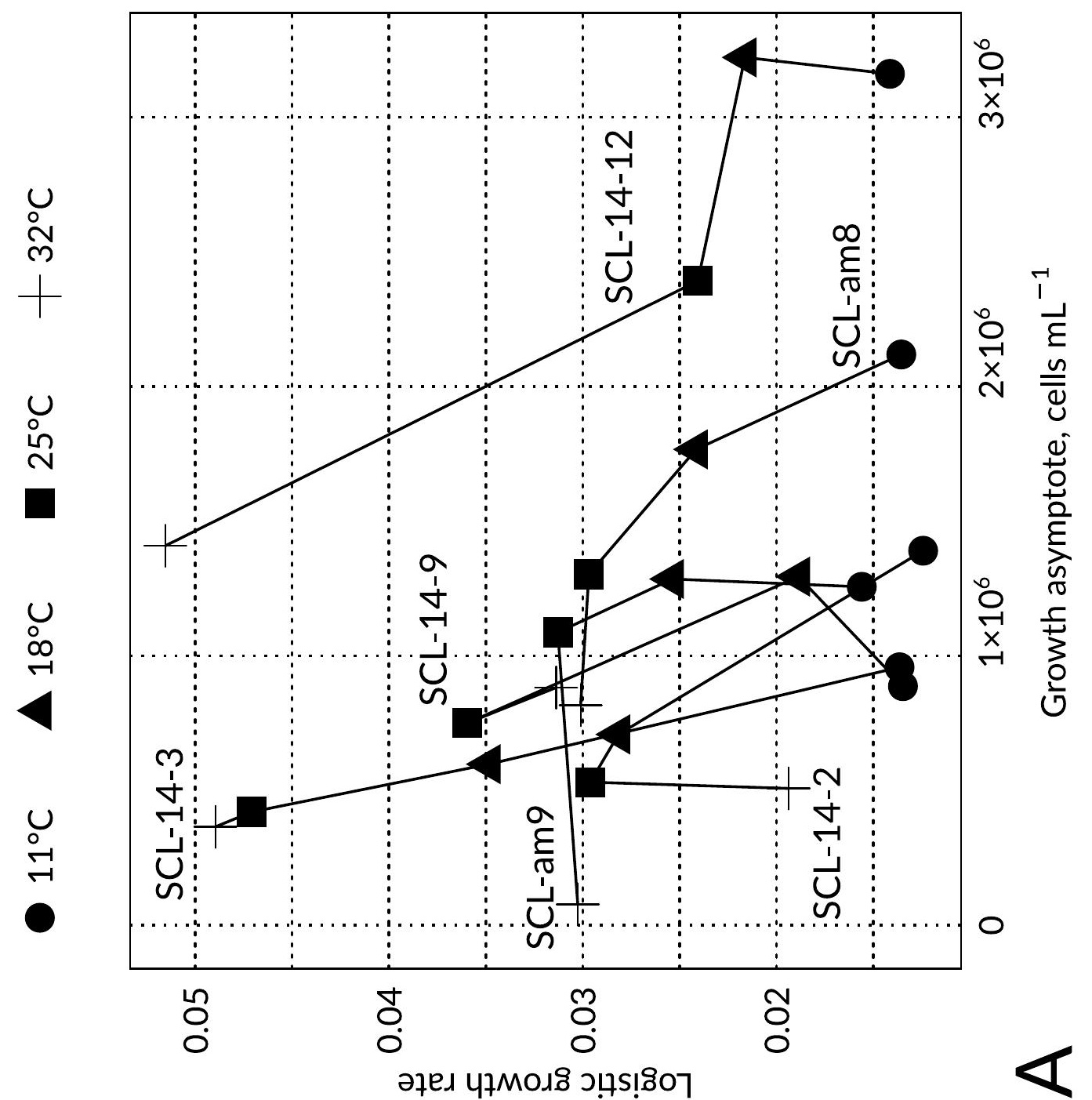


$\infty \Upsilon$

$\stackrel{\sim}{\rightarrow} \sim \cdots$

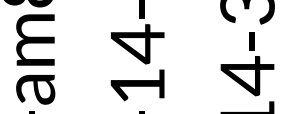

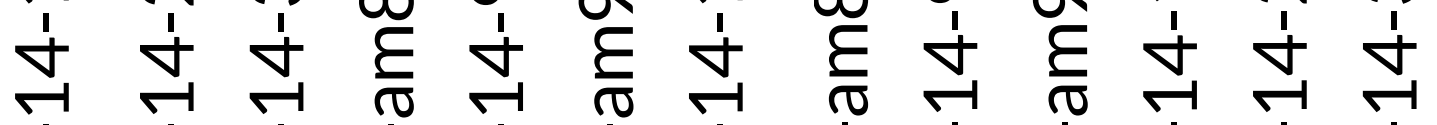

$\stackrel{1}{\cup} \stackrel{1}{\cup} \frac{1}{4}$

ڤे

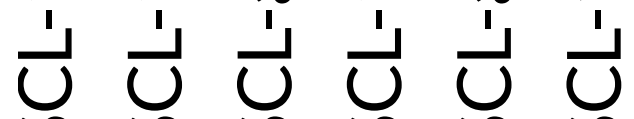

ज $थ$ w

(

守

范

 స 岂
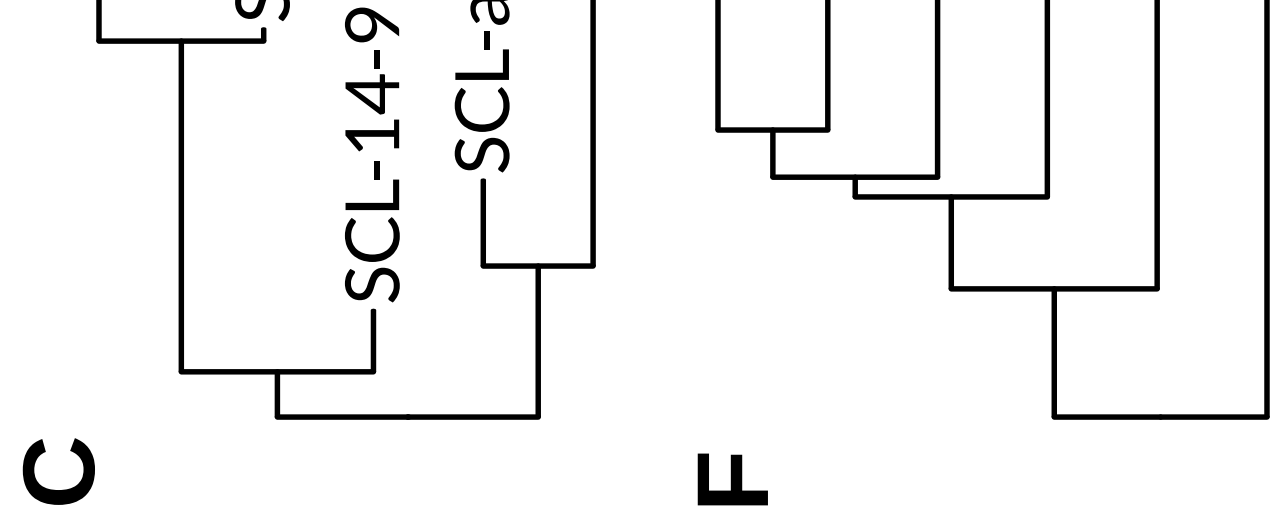

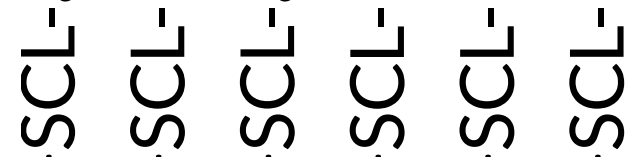

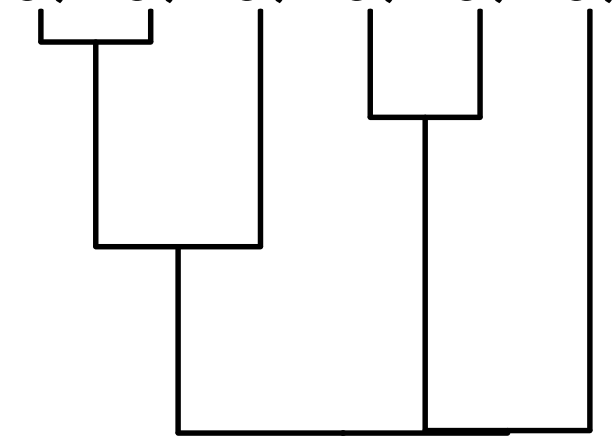

$\square$

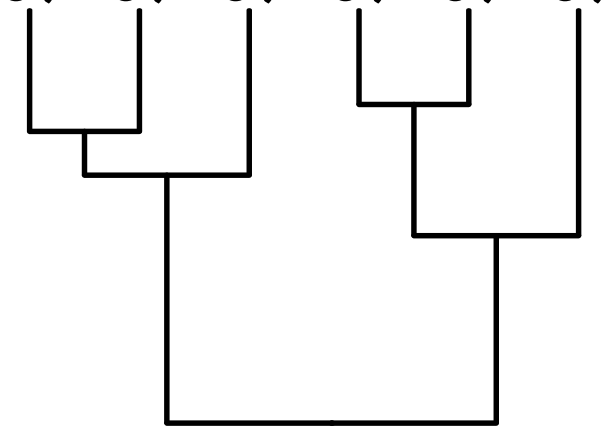

$m$

Ш

I

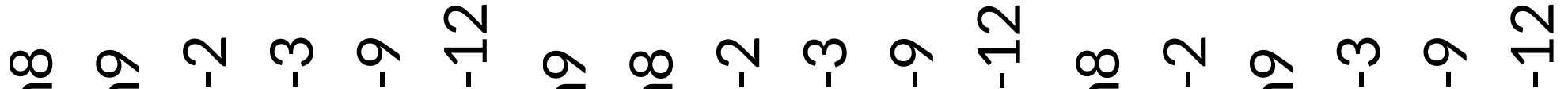

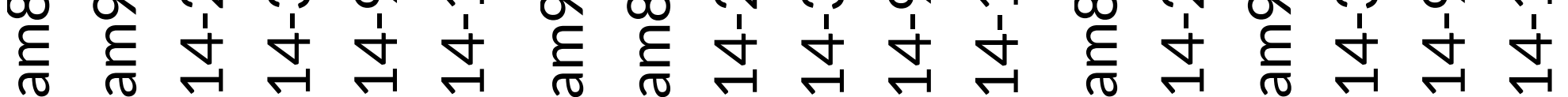

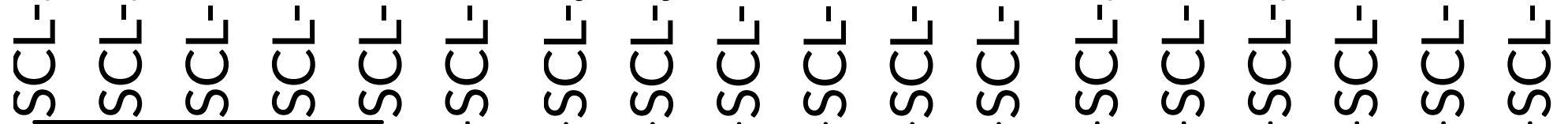
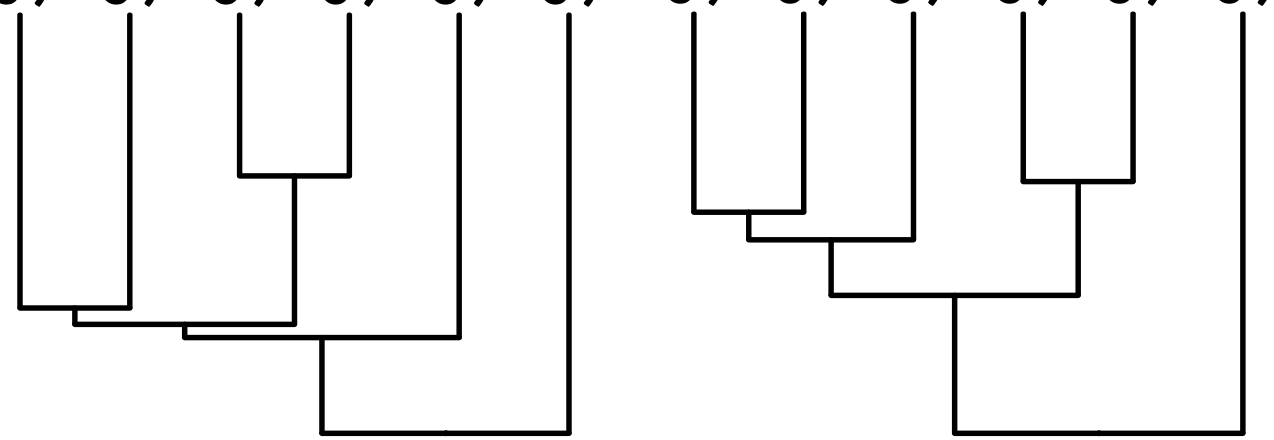
Table 1

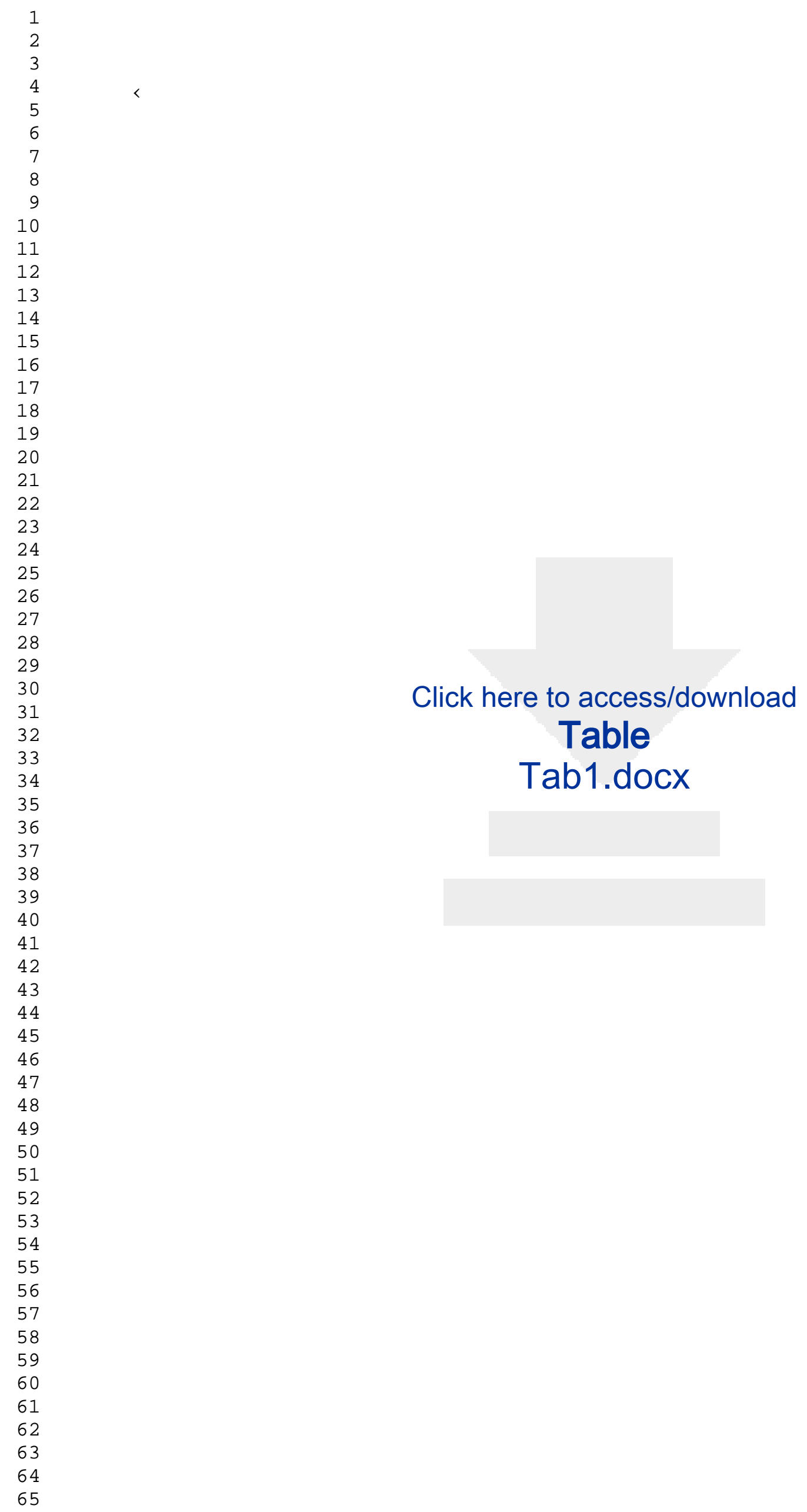


dí\ ÿSupplementaryPlaceholder R $\ddot{y} \ddot{y} \quad$ Ë̈lé 
Table 2

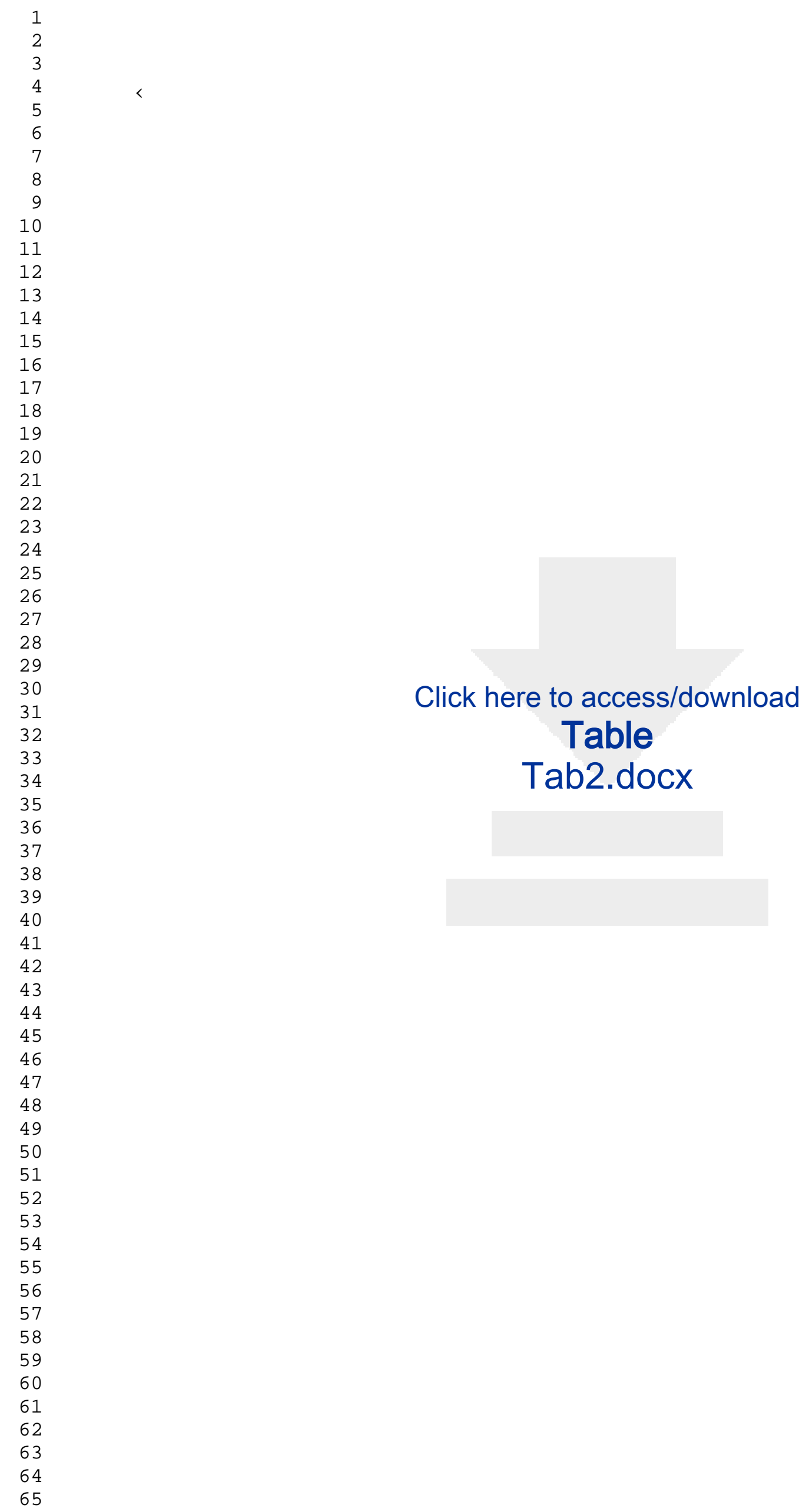


dí\ ÿSupplementaryPlaceholder R $\ddot{y} \ddot{y} \quad$ Ë̈lé 
Table 3

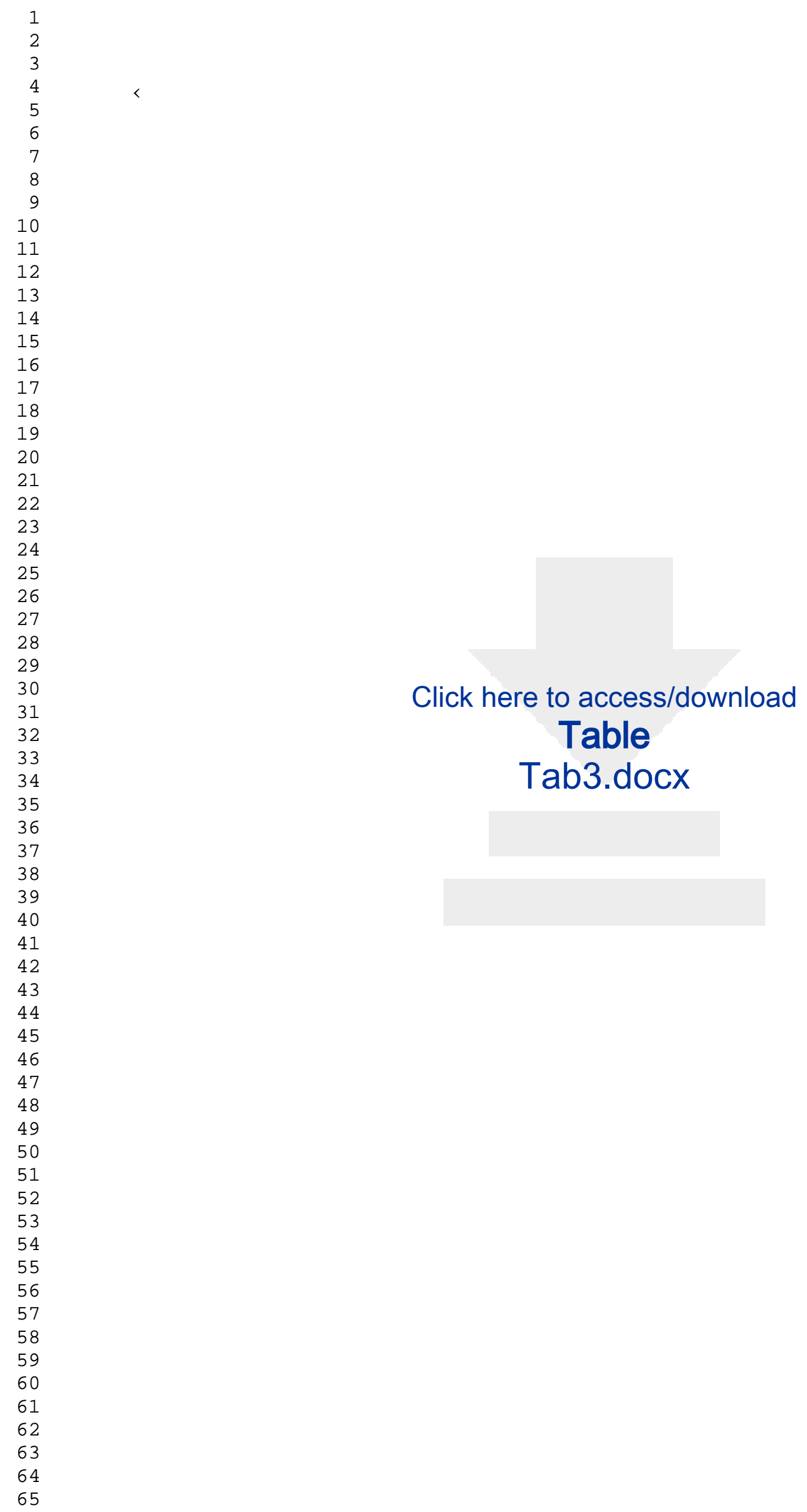


dí\ ÿSupplementaryPlaceholder R $\ddot{y} \ddot{y} \quad$ Ë̈lé 
Table 4

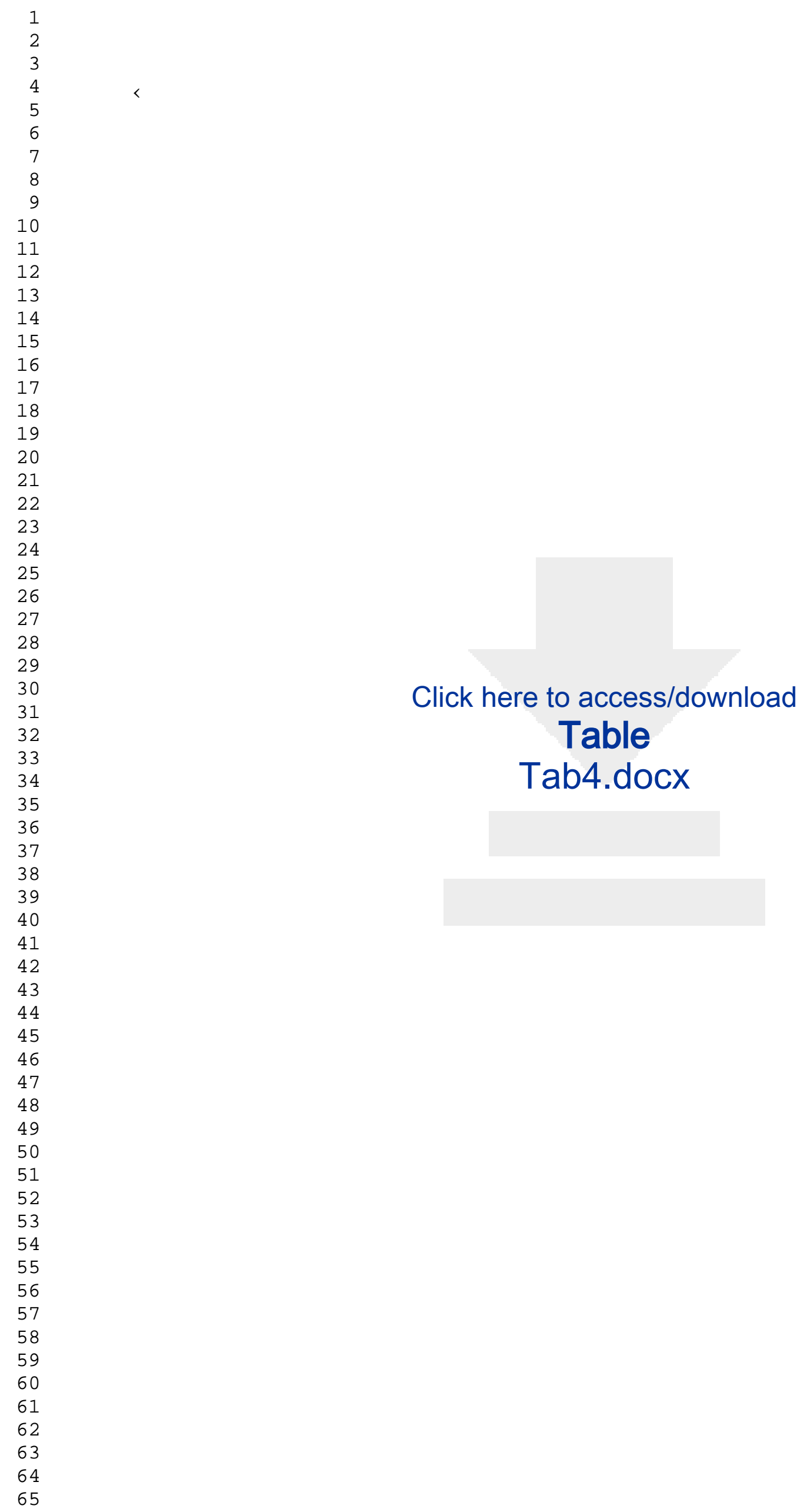

Click here to access/download Tab4.docx 
dí\ ÿSupplementaryPlaceholder R $\ddot{y} \ddot{y} \quad$ Ë̈lé 
Table 5

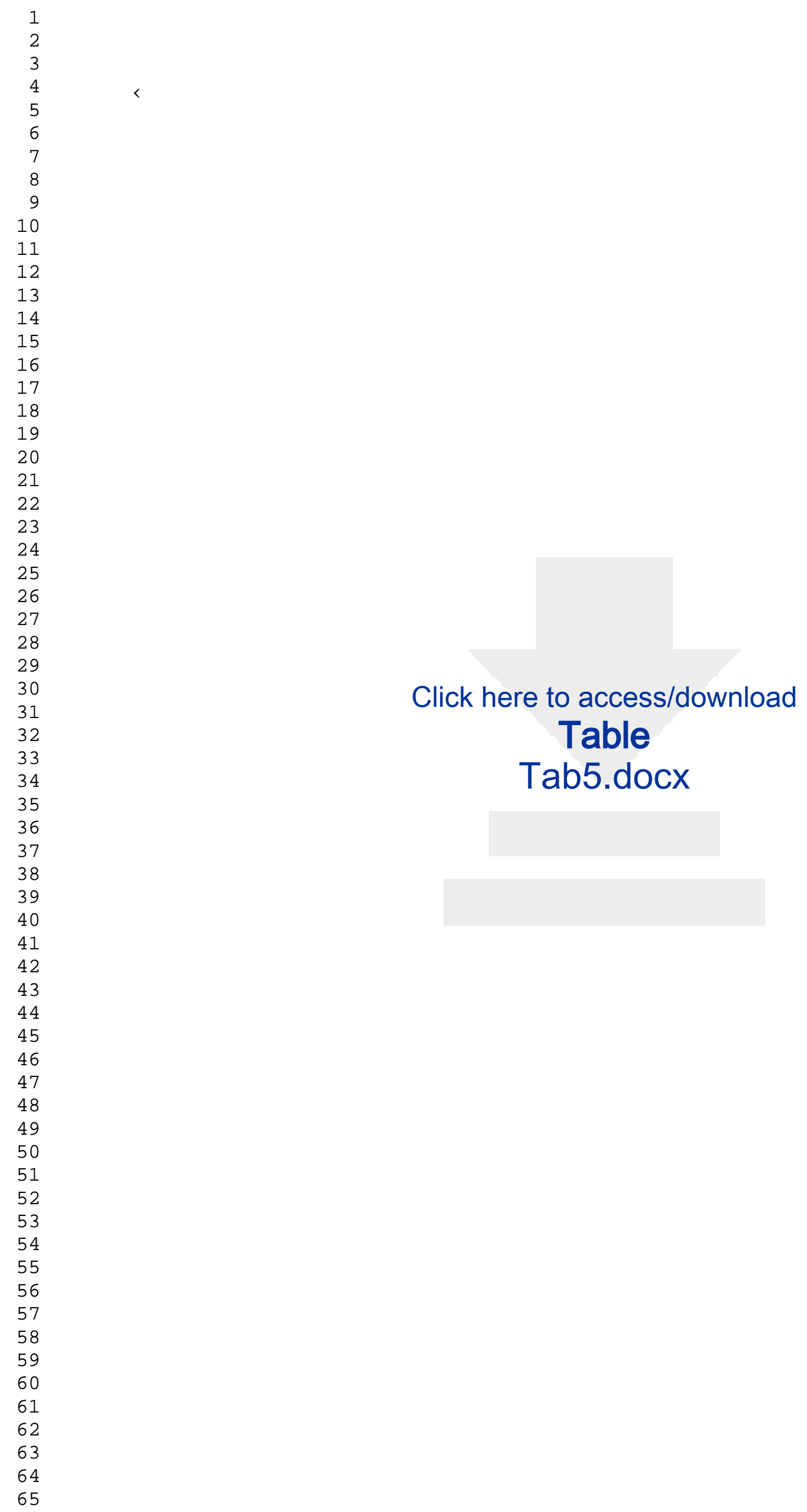


dí\ ÿSupplementaryPlaceholder R $\ddot{y} \ddot{y} \quad$ Ë̈lé 
Click here to access/download e-Component Supplementary material.pdf 